\title{
A Conjugate Heat Transfer Analysis of a Triangular Finned Annulus Based on DG-FEM
}

\author{
Muhammad Ishaq ${ }^{(D)}{ }^{1}$ Khalid Saifullah Syed, ${ }^{2}$ Zafar Iqbal, ${ }^{3}$ and Ahmad Hassan ${ }^{4}{ }^{4}$ \\ ${ }^{1}$ Department of Mathematics, COMSATS University Islamabad (CUI), Vehari Campus, Pakistan \\ ${ }^{2}$ Centre for Advanced Studies in Pure and Applied Mathematics (CASPAM), Bahauddin Zakariya University, Multan, Pakistan \\ ${ }^{3}$ Department of Mathematics, Government Emerson College, Multan, Pakistan \\ ${ }^{4}$ Department of Basic Sciences \& Humanities, University College of Engineering \& Technology, \\ Bahauddin Zakariya University Multan, Pakistan
}

Correspondence should be addressed to Muhammad Ishaq; mishaq151@hotmail.com

Received 28 February 2018; Accepted 14 June 2018; Published 11 July 2018

Academic Editor: Filippo de Monte

\begin{abstract}
Copyright (c) 2018 Muhammad Ishaq et al. This is an open access article distributed under the Creative Commons Attribution License, which permits unrestricted use, distribution, and reproduction in any medium, provided the original work is properly cited.
\end{abstract}

\begin{abstract}
A DG-FEM based numerical investigation has been performed to explore the influence of the various geometric configurations on the thermal performance of the conjugate heat transfer analysis in the triangular finned double pipe heat exchanger. The computed results dictate that Nusselt number in general rises with values of the conductivity ratio of solid and fluid, for the specific configuration parameters considered here. However, the performance of these parameters shows strong influence on the conductivity ratio. Consequently, these parameters must be selected in consideration of the thermal resistance, for better design of heat exchanger.
\end{abstract}

\section{Introduction}

A review of the literature [1-23] of the heat transfer enhancement shows that this is an emergent area. The investigation of the solid-wall conductivities on the heat transfer enhancement in internally finned tube was done by Soliman [1]. Krishen [2] investigated the wall conduction effects on conjugate heat transfer problem in a pipe with finite thickness. Barrozi and Pagliarini [3] showed the conjugate heat transfer results in a circular pipe based on their numerical simulation. Sakakibara et al. [4] performed their analytical study of conjugate heat transfer in the annulus. Their results dictated that conduction has inverse relation to the ratio of the conductivities. Tao [5] performed his work on conjugate heat problem in internally finned tube. One of the outcomes of his study was that heat capacities have stronger influence on heat transfer enhancement. Pagliarini [6] studied the influence of wall conductance in the conjugate problem in the tube. He suggested various limits of the values of wall thickness. A finned double pipe (FDP) heat exchanger, comprising two circular pipes, uses one of the most efficient augmentation techniques in which fins of various shapes are augmented on the inner pipe. Agrawal and Sengupta [7] investigated the heat transfer problem with periodic circular FDP and reported substantial heat transfer rate improvement. Kettner et al. [8] investigated the study of the internally finned tube and recommended that the fin height may be more than $40 \%$ relative to radius of pipe for enhancement of heat with respect to conductivities ratio. Suryanarayana and Apparao [9] reported enhancement of the heat transfer rate in FDP. Fiebig et al. $[10,11]$ did their investigation of heat reversal occurrence and its avoidance in a finned tube. A review of FDP and multitube was presented by Taborek [12]. Syed [13] numerically investigated the heat transfer enhancement in the rectangular FDP. He investigated the influence of various parameters of the configuration of the FDP on the heat transfer characteristics. While investigating the relative performance of the two heated surfaces, he showed that the fin surface is highly effective compared with the inner pipe surface in promoting the convective heat transfer. Dorfman and Renner [14] presented their review on the conjugate problems on heat transfer. They discussed the analytical 
solutions of such problems. An investigation based on the finite element method for the study of the fluid and the heat transfer characteristics in the triangular FDP heat exchanger with fins of equal height was presented by Syed et al. [15]. A uniform heat flux boundary condition (H1) was imposed at the inner pipe. The comparison of triangular and rectangular fins shows that former fin is low in weight compared to latter due to thin top and thus results in low cost. Due to the complexity and mathematical convenience, in the heat transfer problem, some assumptions were always made relax so that the problem may be explored easily. The assumptions of infinitesimal inner pipe wall thickness and infinite thermal conductivity were considered in [15]. Their investigation presented that the Nusselt number is enhanced more than four times as a result of augmentation of triangular fins. Iqbal et al. [16] studied the parabolic FDP and suggested many optimal configurations of FDP based on the Nusselt number. Ishaq et al. [17] studied the heat transfer enhancement in FDP with different fin heights of triangular shape. They showed that a group of triangular fins with unequal heights perform better than the equal heights. Iqbal et al. [18] proposed many optimal fin shapes in FDP. Salman et al. [19] numerically presented the heat transfer enhancement in a tube induced by elliptic-cut and classical twist tape (ECT). Their results showed that the performance of ECT is better than classical twist tape. Iqbal et al. [21] reported many optimum FDP configurations using genetic algorithm based on the weight, heat quantity, cost, and structural integrity of FDP. Syed et al. [22] numerically investigated FDP with variable thickness of the tip of the triangular fins. Their results recommended that the tip thickness may be considered in the designing of FDP. Waseem et al. [23] presented the performance of exponential FDP numerically. They found that Nusselt number may have significant effect on the conductivities ratio values of solid and fluid.

In the present investigation, problem [15] is enhanced by taking into account the assumptions of finite thickness of inner pipe wall of FDP and finite thermal conductivity in the inner pipe wall-fin assembly, carried out in chapter 6 of Ph.D. thesis of corresponding author [20]. It may be remembered that in most of the finned annulus fin surface plays a dominant role in heat transfer. Therefore, it is necessary for an accurate solution of the heat transfer problem in FDP to consider fin conduction in wall-fin assembly. Thus, twodimensional energy equation is considered in the triangular fins and inner pipe wall, in the present investigation. In this way, the present problem takes the form of conjugate heat transfer analysis by considering the conduction in the solid and the convection in the fluid, simultaneously. At the solidfluid interface of such problems, the temperature and heat flux are considered as continuous functions. In the present work, this condition is implemented successfully. The 2dimensional energy equation in the inner pipe wall and fin will be solved subject to H1-boundary conditions. The effect of this more realistic situation of wall-fin conductance will be investigated on the triangular FDP design by studying the temperature distribution and the Nusselt number with the influence of the variations in the height of the triangular fin
$\left(H^{*}\right)$, the inner pipe wall thickness $(\delta)$, the ratio of radii of both pipes $(\widehat{R})$, and the number of triangular fins $(M)$.

The outline of present work consists of 5 sections. Section 2 deals with the present problem and its mathematical model. The DG-FEM based numerical method is described in Section 3. Sections 4 and 5 are, respectively, devoted to results and discussion, as well as the conclusions.

\section{Problem Statement and Its Mathematical Model}

The cross section of the triangular FDP is drawn in Figure 1(a). FDP consists of two concentric circular pipes with a number of longitudinal triangular fins augmented on the inner pipe. Triangular fins are nonporous, straight, and uniformly distributed around the periphery of the outer surface of inner pipe. Thus, the geometry is described by five parameters: the ratio of radii of both pipes, the number of triangular fins, fin half angle, inner pipe wall thickness, and the triangular fin height. The computational domain is sketched in Figure 1(b).

The analysis of the present investigation is based on the following assumptions: there is a steady, laminar flow of a constant property fluid with fully developed motion in the triangular FDP; the fluid motion is in the axial direction due to pressure gradient only; all body forces and the viscous dissipation are taken to be negligible; the axial conduction in both pipe walls and fluid is assumed to be negligible; a uniform heat flux per unit axial length with circumferentially uniform temperature is considered at every cross section; the conditions of adiabatic and thermal boundary are assumed at the inner surface of the outer pipe and at the inner surface of the inner pipe, respectively; a finite thermal resistance of the material of the inner pipe wall-fin assembly is assumed; the continuity conditions of temperature and heat flux are assumed at interface between fluid and wall-fin assembly, and a finite inner pipe wall thickness is considered.

The objective of the present instigation is that a DGFEM based numerical work is to explore the influence of the numerous geometric configurations on the thermal characteristics of the conjugate heat transfer analysis in the triangular finned double pipe heat exchanger.

To make the model equations dimensionless, the following equations are employed.

$$
\begin{aligned}
U^{*} & =\frac{U}{U_{\max }}, \\
R & =\frac{r}{r_{\mathrm{o}}} \\
\widehat{R} & =\frac{r_{i}}{r_{\mathrm{o}}} \\
R_{1} & =\frac{r_{1}}{r_{\mathrm{o}}} \\
\tau(r) & =\frac{T(r, z)-T_{w}(z)}{\dot{Q}^{\prime} / \lambda_{f}},
\end{aligned}
$$




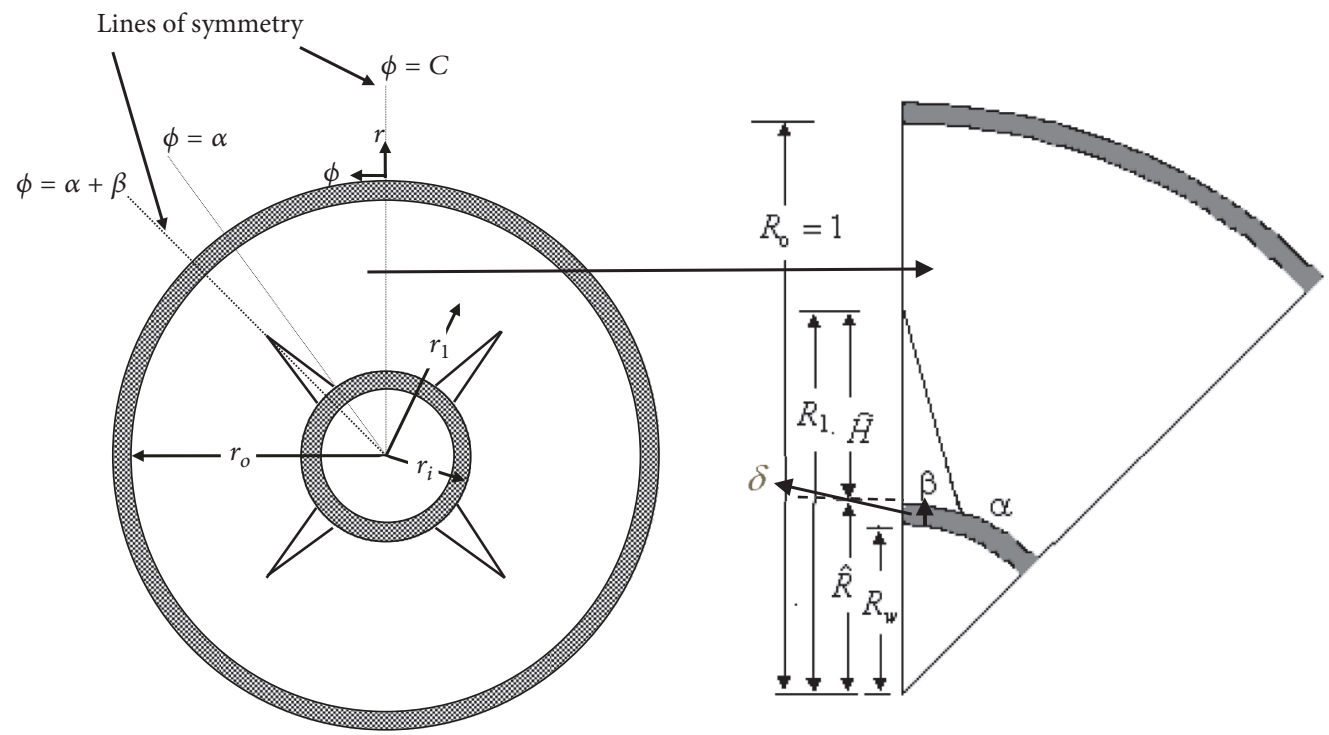

(a)

(b)

Figure 1: (a) Cross section of the triangular FDP. (b) Computational domain.

where $U_{\max }=-(1 / 4 \mu)(d p / d z) r_{\mathrm{o}}^{2}\left\{1-R_{\mathrm{m}}^{2}+2 R_{\mathrm{m}}^{2} \ln R_{\mathrm{m}}\right\}$ and $R_{\mathrm{m}}=r_{\mathrm{m}} / r_{\mathrm{o}}=\sqrt{\left(1-\widehat{R}^{2}\right) / 2 \ln (1 / \widehat{R})}$. Using these variables, the governing equations - momentum, energy, and heat equations-together with the corresponding boundary conditions may be expressed in dimensionless form as in $[15,23]$.

Momentum equation:

$$
\frac{1}{R} \frac{\partial U^{*}}{\partial R}+\frac{\partial^{2} U^{*}}{\partial R^{2}}+\frac{1}{R^{2}} \frac{\partial^{2} U^{*}}{\partial \phi^{2}}=\frac{-4}{C}
$$

where $C=\left(1-R_{\mathrm{m}}^{2}+2 R_{\mathrm{m}}^{2} \ln R_{\mathrm{m}}\right)$.

Boundary conditions:

(i) $U^{*}=0$ at $R=\widehat{R}$ and $0 \leq \phi \leq \alpha$

(ii) $U^{*}=0$

at $R=\frac{R_{1} \widehat{R} \sin \beta}{\widehat{R} \sin (\phi-\alpha)-R_{1} \sin (\phi-\alpha-\beta)}$ and $\alpha \leq \phi \leq \alpha+\beta$

(iii) $U^{*}=0$ at $R=1$ and $0 \leq \phi \leq \alpha+\beta$

(iv) $\frac{\partial U^{*}}{\partial \phi}=0 \quad$ at $\phi=0$ and $\widehat{R} \leq R \leq 1$

(v) $\frac{\partial U^{*}}{\partial \phi}=0 \quad$ at $\phi=\alpha+\beta$ and $R_{1} \leq R \leq 1$

Energy equation:

$$
\frac{\partial^{2} \tau^{f}}{\partial R^{2}}+\frac{1}{R} \frac{\partial \tau^{f}}{\partial R}+\frac{1}{R^{2}} \frac{\partial^{2} \tau^{f}}{\partial \phi^{2}}=\frac{U^{*}}{A_{c}^{*} \bar{U}^{*}}
$$

Heat equation:

$$
\frac{\partial^{2} \tau^{s}}{\partial R^{2}}+\frac{1}{R} \frac{\partial \tau^{s}}{\partial R}+\frac{1}{R^{2}} \frac{\partial^{2} \tau^{s}}{\partial \phi^{2}}=0
$$

Corresponding Boundary conditions are

(i) $\tau^{s}=0$ at $R=R_{w}$ and $0 \leq \phi \leq \alpha+\beta$

(ii) $\frac{\partial \tau^{f}}{\partial R}=0 \quad$ at $R=1$ and $0 \leq \phi \leq \alpha+\beta$

(iii) $\frac{\partial \tau^{s}}{\partial \phi}=0 \quad$ at $\phi=0$ and $R_{w} \leq R \leq \widehat{R}$

(iv) $\frac{\partial \tau^{f}}{\partial \phi}=0 \quad$ at $\phi=0$ and $\widehat{R} \leq R \leq 1$

(v) $\frac{\partial \tau^{s}}{\partial \phi}=0 \quad$ at $\phi=\alpha+\beta$ and $R_{w} \leq R \leq R_{1}$

(vi) $\frac{\partial \tau^{f}}{\partial \phi}=0 \quad$ when $R_{1} \leq R \leq 1$ and $\phi=\alpha+\beta$

To maintain the energy balance at the solid-fluid interface, the following conditions of continuity of the temperature and flux are employed.

$$
\begin{aligned}
& \tau^{s}=\tau^{f} \quad \text { at } R=\widehat{R} \text { and } 0 \leq \phi \leq \alpha \\
& \begin{aligned}
\tau^{s} & =\tau^{f} \\
\text { at } R & =\frac{R_{1} \widehat{R} \sin \beta}{\widehat{R} \sin (\phi-\alpha)-R_{1} \sin (\phi-\alpha-\beta)} \text { and } \alpha \leq \phi \leq \alpha+\beta \\
\frac{\partial \tau^{s}}{\partial R} & =\frac{1}{\Omega} \frac{\partial \tau^{f}}{\partial R} \quad \text { at } R=\widehat{R} \text { and } 0 \leq \phi \leq \alpha \\
\frac{\partial \tau^{s}}{\partial \phi} & =\frac{1}{\Omega} \frac{\partial \tau^{f}}{\partial \phi}
\end{aligned}
\end{aligned}
$$

at $R=\frac{R_{1} \widehat{R} \sin \beta}{\widehat{R} \sin (\phi-\alpha)-R_{1} \sin (\phi-\alpha-\beta)}$ and $\alpha \leq \phi \leq \alpha+\beta$, 
where $\Omega=\lambda_{s} / \lambda_{f}$ is the thermal conductivity ratio of the inner pipe wall-fin assembly and the fluid; $\tau^{s}$ and $\tau^{f}$ denote temperature in the inner pipe wall and triangular fin assembly and the fluid, respectively.

\section{DG-FEM Based Numerical Method}

The higher order discontinuous Galerkin finite element method (DG-FEM) is chosen to compute the solution of the present conjugate heat transfer problem in the triangular FDP. DG-FEM possesses the characteristics of finite volume method (FVM) and finite element method (FEM). In DGFEM, the solution is assumed to be discontinuous over the local elemental boundaries instead of continuous as in the case of the classical FEM. The numerical fluxes are used to tackle this discontinuity across the elemental faces [24]. DGFEM based on [24] is employed to compute the solution of the conjugate heat transfer in triangular FDP.

The DG-FEM formulation of the governing equations is described below.

The momentum equation may be written as

$$
\nabla^{2} U^{*}=f
$$

where $f=-4 / C$. This equation is equivalent to the following system of first-order partial differential equations:

$$
\begin{aligned}
\nabla \cdot \mathbf{q} & =f(\mathbf{x}), \\
\nabla u & =\mathbf{q} .
\end{aligned}
$$

Using $\mathbf{q}$ in its components form in the above equation, the resulting first-order system is

$$
\begin{aligned}
q^{x} & =\frac{\partial u}{\partial x}, \\
q^{y} & =\frac{\partial u}{\partial y} \\
\frac{\partial q^{x}}{\partial x}+\frac{\partial q^{y}}{\partial y} & =f(u) .
\end{aligned}
$$

The domain $\Omega^{d}$ is divided into $K$ nonoverlapping triangular mesh $\Omega_{k}^{d}$ with condition

$$
\Omega^{d}=\bigcup_{k=1}^{K} \Omega_{k}^{d}
$$

The solution in each subdomain $\Omega_{k}^{d}$ is well approximated by the local polynomial of degree $N$ :

$$
\begin{aligned}
{\left[\begin{array}{c}
u^{k}(\mathbf{x}) \\
\mathbf{q}^{k}(\mathbf{x})
\end{array}\right] } & \cong\left[\begin{array}{c}
u_{h}^{k}(\mathbf{x}) \\
\mathbf{q}_{h}^{k}(\mathbf{x})
\end{array}\right]=\sum_{i=1}^{N p}\left[\begin{array}{c}
u_{h}^{k}\left(\mathbf{x}_{i}^{k}\right) \\
\mathbf{q}_{h}^{k}\left(\mathbf{x}_{i}^{k}\right)
\end{array}\right] \ell_{i}^{k}(\mathbf{x}) \\
& =\sum_{i=1}^{N p}\left[\begin{array}{c}
u_{i}^{k} \\
\mathbf{q}_{i}^{k}
\end{array}\right] \ell_{i}^{k}(\mathbf{x}),
\end{aligned}
$$

and $f(u)$ is

$$
f^{k}\left(u^{k}\right) \cong f_{h}^{k}\left(u_{h}^{k}\right)=\sum_{i=1}^{N_{p}} f_{h}^{k}\left(\mathbf{x}_{i}^{k}\right) \ell_{i}^{k}(\mathbf{x})=\sum_{i=1}^{N_{p}} f_{i}^{k} \ell_{i}^{k}(\mathbf{x}),
$$

where $\mathbf{x}_{i}^{k}$ are the $N_{p}=(N+1)(N+2) / 2$ grid points in the $k$ th element and $\ell_{i}^{k}(\mathbf{x})$ is the $2 \mathrm{D}$ Lagrange polynomial based on these points.

The local element approximation of (9)-(10) may be written as

$$
\begin{array}{r}
q_{h}^{x k}=\partial_{x} u_{h}^{k} \\
q_{h}^{y k}=\partial_{y} u_{h}^{k} \\
\partial_{x} q_{h}^{x k}+\partial_{y} q_{h}^{y k}=f\left(u_{h}^{k}\right)
\end{array}
$$

Eq. (14) is first multiplied with test functions $\ell_{i}^{k}(\mathbf{x})$, followed by integrating by parts twice over the element $\Omega_{k}^{d}$, to obtian the following strong form.

$$
\begin{aligned}
& \int_{\Omega_{k}^{d}} q_{h}^{x k} \ell_{i}^{k}(\mathbf{x}) d \mathbf{x}=\int_{\Omega_{k}^{d}} \ell_{i}^{k}(\mathbf{x}) \partial_{x} u_{h}^{k} d \mathbf{x}-\int_{\partial \Omega_{k}^{d}}\left(\widehat{n}_{x}\right. \\
& \left.\cdot\left(u_{h}^{k}-\left(u_{h}^{k}\right)^{*}\right)\right) \ell_{i}^{k}(\mathbf{x}) d \mathbf{x} \\
& \int_{\Omega_{k}^{d}} q_{h}^{y k} \ell_{i}^{k}(\mathbf{x}) d \mathbf{x}=\int_{\Omega_{k}^{d}} \ell_{i}^{k}(\mathbf{x}) \partial_{y} u_{h}^{k} d \mathbf{x}-\int_{\partial \Omega_{k}^{d}}\left(\widehat { n } _ { y } \cdot \left(u_{h}^{k}\right.\right. \\
& \left.\quad-\left(u_{h}^{k}\right)^{*}\right) \ell_{i}^{k}(\mathbf{x}) d \mathbf{x} \\
& \int_{\Omega_{k}^{d}}\left(\partial_{x} q_{h}^{x k}+\partial_{y} q_{h}^{y k}\right) \ell_{i}^{k}(\mathbf{x}) d \mathbf{x}-\int_{\partial \Omega_{k}^{d}}\left(\widehat{n}_{x}\right. \\
& \left.\cdot\left(q_{h}^{x k}-\left(q_{h}^{x k}\right)^{*}\right)+\widehat{n}_{y} \cdot\left(q_{h}^{y k}-\left(q_{h}^{y k}\right)^{*}\right)\right) \ell_{i}^{k}(\mathbf{x}) d \mathbf{x} \\
& =\int_{\Omega_{k}^{d}} f\left(u_{h}^{k}\right) \ell_{i}^{k}(\mathbf{x}) d \mathbf{x}
\end{aligned}
$$

Using (12)-(13) in (15)-(17), the following system of differential algebraic equations is obtained.

$$
\begin{gathered}
M^{k} \mathbf{q}_{h}^{x k}=S^{x} \mathbf{u}_{h}^{k}-\int_{\partial \Omega_{k}^{d}}\left(\widehat{n}_{x} \cdot\left(u_{h}^{k}-\left(u_{h}^{k}\right)^{*}\right)\right) L_{h}^{k}(\mathbf{x}) d \mathbf{x} \\
M^{k} \mathbf{q}_{h}^{y k}=S^{y} \mathbf{u}_{h}^{k}-\int_{\partial \Omega_{k}^{d}}\left(\widehat{n}_{y} \cdot\left(u_{h}^{k}-\left(u_{h}^{k}\right)^{*}\right)\right) L_{h}^{k}(\mathbf{x}) d \mathbf{x} \\
S^{x} \mathbf{q}_{h}^{x k}+S^{y} \mathbf{q}_{h}^{y k}-\int_{\partial \Omega_{k}^{d}}\left(\widehat{n}_{x} \cdot\left(q_{h}^{x k}-\left(q_{h}^{x k}\right)^{*}\right)+\widehat{n}_{y}\right. \\
\left.\cdot\left(q_{h}^{y k}-\left(q_{h}^{y k}\right)^{*}\right)\right) L_{h}^{k}(\mathbf{x}) d \mathbf{x}=M^{k} \mathbf{f}_{h}^{k},
\end{gathered}
$$

where $M_{i j}^{k}=\int_{\Omega_{k}^{d}} \ell_{i}^{k}(\mathbf{x}) \ell_{j}^{k}(\mathbf{x}) d \mathbf{x}$ is the mass matrix; $S_{i j}^{x}=$ $\int_{\Omega_{k}^{d}} \ell_{i}^{k}(\mathbf{x}) \partial_{x} \ell_{j}^{k}(\mathbf{x}) d \mathbf{x}$ and $S_{i j}^{y}=\int_{\Omega_{k}^{d}} \ell_{i}^{k}(\mathbf{x}) \partial_{y} \ell_{j}^{k}(\mathbf{x}) d \mathbf{x}$ are the stiffness matrices. 
Similarly, the strong form of the energy equation can be derived using $\nabla \tau^{f^{*}}={ }^{\tau^{f}} \mathbf{q}$, as

$$
\begin{gathered}
S^{x \tau^{f}} \mathbf{q}_{h}^{x k}+S^{y \tau^{f}} \mathbf{q}_{h}^{y k}-\int_{\partial \Omega_{k}^{d}}\left(\widehat{n}_{x} \cdot\left(\tau^{f} q_{h}^{x k}-\left(\tau^{f} q_{h}^{x k}\right)^{*}\right)\right. \\
\left.+\widehat{n}_{y} \cdot\left(\tau^{f} q_{h}^{y k}-\left(\tau^{f} q_{h}^{y k}\right)^{*}\right)\right) L_{h}^{k}(\mathbf{x}) d \mathbf{x}=M^{k} \mathbf{g}_{h}^{k} \\
M^{k \tau^{f}} \mathbf{q}_{h}^{x k}=S^{x} \tau_{h}^{* k}-\int_{\partial \Omega_{k}^{d}}\left(\widehat{n}_{x} \cdot\left(\tau_{h}^{* k}-\left(\tau_{h}^{* k}\right)^{*}\right)\right) \\
\cdot L_{h}^{k}(\mathbf{x}) d \mathbf{x} \\
M^{k \tau^{f}} \mathbf{q}_{h}^{y k}=S^{y} \tau_{h}^{* k}-\int_{\partial \Omega_{k}^{d}}\left(\widehat{n}_{y} \cdot\left(\tau_{h}^{* k}-\left(\tau_{h}^{* k}\right)^{*}\right)\right) \\
\cdot L_{h}^{k}(\mathbf{x}) d \mathbf{x}
\end{gathered}
$$

where $g=U^{*} / A_{c}^{*} \bar{U}^{*}$.

In the same way, the strong form of the heat equation may be derived using $\nabla \tau^{s *}={ }^{\tau^{s}} \mathbf{q}$, as

$$
\begin{gathered}
S^{x \tau^{s}} \mathbf{q}_{h}^{x k}+S^{y \tau^{s}} \mathbf{q}_{h}^{y k}-\int_{\partial \Omega_{k}^{d}}\left(\widehat{n}_{x} \cdot\left(\tau^{s} q_{h}^{x k}-\left(\tau^{s} q_{h}^{x k}\right)^{*}\right)\right. \\
\left.+\widehat{n}_{y} \cdot\left(\tau^{s} q_{h}^{y k}-\left(\tau^{s} q_{h}^{y k}\right)^{*}\right)\right) L_{h}^{k}(\mathbf{x}) d \mathbf{x}=0 \\
M^{k \tau^{s}} \mathbf{q}_{h}^{x k}=S^{x} \tau_{h}^{* k}-\int_{\partial \Omega_{k}^{d}}\left(\widehat{n}_{x} \cdot\left(\tau_{h}^{* k}-\left(\tau_{h}^{* k}\right)^{*}\right)\right) \\
\cdot L_{h}^{k}(\mathbf{x}) d \mathbf{x} \\
M^{k \tau^{s}} \mathbf{q}_{h}^{y k}=S^{y} \tau_{h}^{* k}-\int_{\partial \Omega_{k}^{d}}\left(\widehat{n}_{y} \cdot\left(\tau_{h}^{* k}-\left(\tau_{h}^{* k}\right)^{*}\right)\right) \\
\cdot L_{h}^{k}(\mathbf{x}) d \mathbf{x}
\end{gathered}
$$

In [17], they proposed 2nd-order local polynomial approximation on the coarse mesh for DG-FEM and the same is employed here. The steps of algorithm employed in the present investigation are as follows: Initial (coarser) mesh is generated in the domain (Figure 1(b)); first the fluid region mesh is extracted and then momentum equation in it is solved; and in the last step, both the heat and energy equations are simultaneously solved in the whole domain. Due to the approximation of Riemann's problem by stabilized internal penalty flux in DG-FEM, the conditions of continuity at the fluid-solid interface are weakly imposed, automatically $[23,24]$. If the temperature gradient $\left(q_{n}\right)$ is along the normal, then the interface conditions (6i) and (6j) may be expressed as

$$
\begin{aligned}
& q_{x}^{s}=\frac{1}{\Omega} q_{x}^{f} \\
& q_{y}^{s}=\frac{1}{\Omega} q_{y}^{f},
\end{aligned}
$$

where $q_{x}^{f}=\partial T^{f} / \partial x, q_{x}^{s}=\partial T^{s} / \partial x, q_{y}^{f}=\partial T^{f} / \partial y$, and $q_{y}^{s}=$ $\partial T^{s} / \partial y$.
It may be noted that there are two values of the field variables: interior (-) and exterior (+), across the edge of an element in DG-FEM. Therefore, one should impose fluid side fluxes as interior and the solid side fluxes as exterior fluxes for the solution of energy equation in fluid region and to find solution of the heat equation in the solid region, the case is vice versa. Thus, these are implemented as

$$
\begin{aligned}
& q_{x}^{s,-}=q_{x}^{s}, \\
& q_{x}^{s,+}=\frac{1}{\Omega} q_{x}^{f,+} \\
& q_{x}^{f,-}=q_{x}^{f}, \\
& q_{x}^{f,+}=\Omega q_{x}^{s,+} \\
& d q_{x}^{f}=q_{x}^{-}-q_{x}^{+}=q_{x}^{f}-\Omega q_{x}^{s} \\
& d q_{x}^{s}=q_{x}^{-}-q_{x}^{+}=q_{x}^{s}-\frac{1}{\Omega} q_{x}^{f} \\
& q_{y}^{s,-}=q_{y}^{s}, \\
& q_{y}^{s,+}=\frac{1}{\Omega} q_{y}^{f,+} \\
& q_{y}^{f,-}=q_{y}^{f}, \\
& q_{y}^{f,+}=\Omega q_{y}^{s,+} \\
& d q_{y}^{f}=q_{y}^{-}-q_{y}^{+}=q_{y}^{f}-\Omega q_{y}^{s} \\
& d q_{y}^{s}=q_{y}^{-}-q_{y}^{+}=q_{y}^{s}-\frac{1}{\Omega} q_{y}^{f}
\end{aligned}
$$

3.1. Validation of Computed Results. The validation of computational procedure and its computer implementation are carried out by comparing the numerical solution and the exact solution of the nonconjugate problem of laminar flow on the shell side of unfinned double pipe. The products of the Fanning friction factor and the Reynolds number $f$ Re and the Nusselt number $\overline{N u}$ are used as performance measures. These quantities have been computed for various values of $\widehat{R}$. The maximum percentage errors in these quantities are, respectively, $0.06 \%$ and $0.15 \%$. Another source of validation of the present results is their comparison with those of [15] when the conductive resistance in the wall-fin assembly is taken to be negligible. This is to note that the results of [15] were obtained using the finite element method with $h$-adaptivity. The comparison is shown in Figure 2.

\section{Results and Discussion}

The present work is aimed at investigating the overall thermal performance, in terms of fully developed $\overline{\mathrm{Nu}}$ of the triangular FDP heat exchanger when the thermal conductivity and inner pipe wall thickness are varied as parameters. Other parameters to be investigated are the design parameters, the fin height $H^{*}$, the number of fins $M$, the ratio of radii 


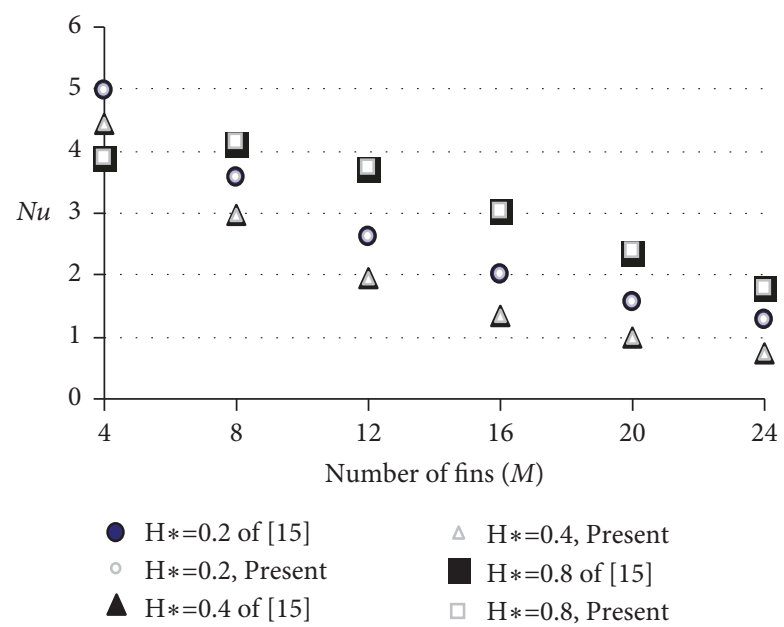

Figure 2: Comparison of the results of $\overline{N u}$ based on the hydraulic diameter against $M$ at $\widehat{R}=0.25, \beta=3^{\circ}$, and $\delta=0.05$ with those of [15].

$\widehat{R}$, and the fin thickness $\beta$, so that a better design for the present more realistic situation may be reached as compared to that given in [15]. The inner pipe wall thickness is made dimensionless as by the relation $\delta=\left(r_{i}-r_{w}\right) / r_{o}$. The values of the above stated parameters taken in the present work are $H^{*}=\{0.2,0.4,0.6,0.8\}, \beta=\left\{3^{\circ}, 5^{\circ}\right\}, \widehat{R}=\{0.25,0.5,0.6\}, \delta=$ $\{0.05,0.1,0.2\}, \Omega=\{1,2,10,20,50,100,500,1000,5000, \infty\}$, and $M=\{4,8,12,16,20,24\}$. Here $\Omega=\infty$ represents the case of [15].

To understand the physical performance of the present problem, the velocity and temperature contours are presented in Figure 3 for the specified parameters $\beta=3^{\circ}, 5^{\circ}, \widehat{R}=$ $0.25,0.5, M=12,18, H^{*}=0.6,0.8$, and $\Omega$ varying from 1 to $\infty$, where the velocity field is normalized by the maximum velocity so that its values lie between 0 and 1 and the temperature field $\tau^{*}$, being a normalized difference of fluid and wall temperatures, has smaller values in the regions of higher temperature and larger values in the lower temperature zones.

Figures 3(a)-3(d)(i) present the velocity contours for various geometric parameters mentioned in the corresponding figure. The observations/comments like large velocity gradients, high velocity zone, formation of one-dimensional flow, stagnant fluid at wall-fin assembly, and the effects of increasing fin height as well as number of fins, as mentioned in $[15,23]$, can easily be observed in these figures. It may be noted again that the results of [15] are based on FEM. The effects of $\delta$ are not significant. Moreover, the zero velocity at the outer surface of inner pipe, the inner surface of the outer pipe, and the fin surface confirms the validity of the present model and its numerical implementation.

Figures 3(a)-3(d)(ii) show the isotherms of the case when the material of the inner pipe, fin, and fluid is same; i.e., conductivity ratio $(\Omega)$ is one. One can easily observe that the isotherms in the inner pipe, in the fluid area that is in the vicinity of outside surface of inner pipe and in the triangular fins, are in circular shape, represent heat transfer process at equal rate, and confirm the validity of the physical model and numerical method. As the values of $\Omega$ rise from 1 to $\infty$ relative to the fluid, the circular isotherms start changing its behavior as shown in Figures 3(a)-3(d)(iii-xii). The temperature contours tend to become straight between two consecutive fins. This isotherm behavior is matchable as in case of exponential fin [23]. Its means that the temperature gradient in this region is increasing with increase of the conductivity of the inner pipe and fin material. It is also clear that the rate of heat transfer at fluid-fin interface is more than that of inner pipe-fluid interface. It may be interesting to note that, with rising the value of $\Omega$, the heat is transferred quickly at the solid-fluid interface, which is the cause of larger temperature differences and hence plays its significant role in promoting the heat transfer coefficient.

As described earlier, $\overline{N u}$ is used as a thermal performance measure of the triangular FDP heat exchanger. It is defined below based on the two diameter values: hydraulic and equivalent.

$$
\begin{gathered}
\overline{N u}=\frac{D_{H}^{*}}{p_{h}^{*} \tau_{b}^{*}}, \\
{\overline{N u_{e}}}=\frac{D_{e}^{*}}{p_{e}^{*} \tau_{b}^{*}},
\end{gathered}
$$

The terms $p_{h}^{*}, D_{H}^{*}, D_{e}^{*}, \tau_{b}^{*}$, and $p_{e}^{*}$ are described in nomenclature.

$\overline{N u}$ is plotted against the ratio of conductivities $(\Omega)$ at $\widehat{R}=0.25, \beta=3^{\circ}, H^{*}=0.4$ and $0.8, \delta=0.05$ and 0.2 , and $4 \leq M \leq 24$, as presented in Figure 4. The effect of the rising values of $\Omega$ on $\overline{N u}$ is strongly prominent which shows the increasing trend of $\overline{N u}$ for $4 \leq M \leq 24$ with $40 \%$ of fin height as depicted in Figures 4(a) and 4(b). This trend validates the previous observations of quick heat transfer at the fluid-solid interface, higher temperature differences, and promoting heat transfer coefficient due to higher values of $\Omega$. It may also be noted from these figures that, at fixed chosen value of $\Omega$, the trend of $\overline{N u}$ curves is decreasing with increase of $M$. However, due to increase in the number of fins, these curves get closer and in turn deteriorate the rate of $\overline{\mathrm{Nu}}$ decrease. Moreover, as the value of $\Omega$ is increased, the $\overline{N u}$ curves corresponding to various number of fins get wider apart, showing the more significant effect of $M$ for higher values of the conductivity ratio. A similar trend of $\overline{N u}$ curves may be observed in Figures 4(c) and 4(d) for the case when the triangular fins have $80 \%$ height except the case of $\overline{N u}$ curves at about $\Omega \approx 1000$, in which $\overline{N u}$ curves for $M=8$ are overriding the curves for $M=4$. It may also be observed in comparing Figures $4(\mathrm{a}) / 4$ (c) to Figures $4(\mathrm{~b}) / 4$ (d) that, with increase of the thickness of the inner pipe wall, at the lower values of $\Omega$ the $\overline{N u}$ curves get closer and in turn reduce the values of $\overline{N u}$. Thus, the effect of inner pipe wall is more prominent only for lower values of the conductivity ratio. This trend also supports the present work that the thicker inner pipe walls offer more resistance in heat conduction at smaller $\Omega$ and thus reduce the values of heat transfer coefficient. 


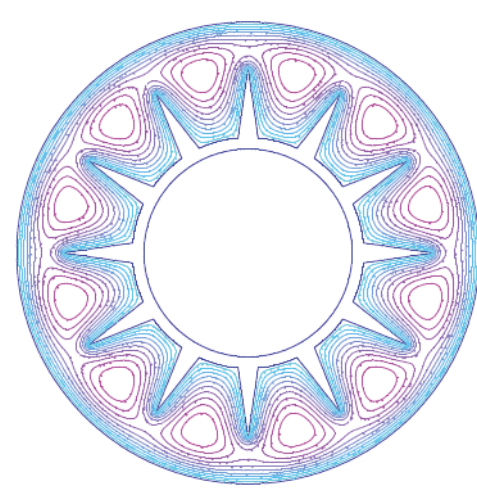

(i)

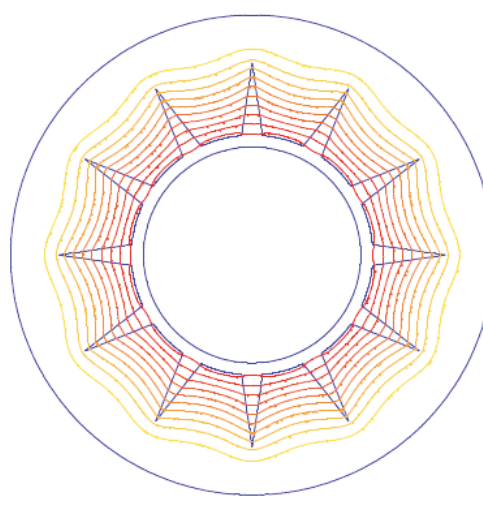

(iv) $\Omega=5$

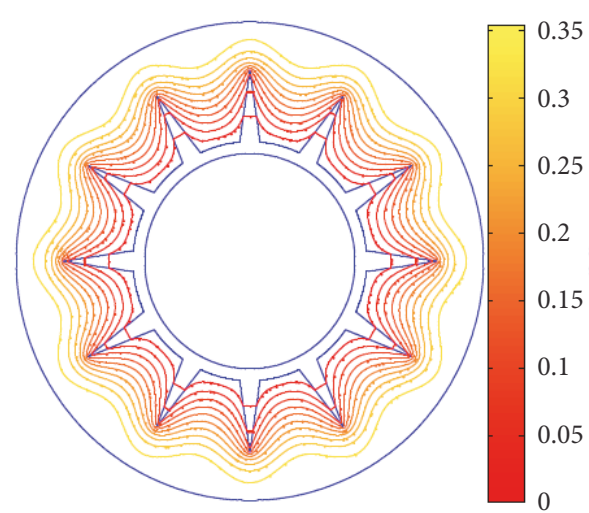

(vii) $\Omega=50$

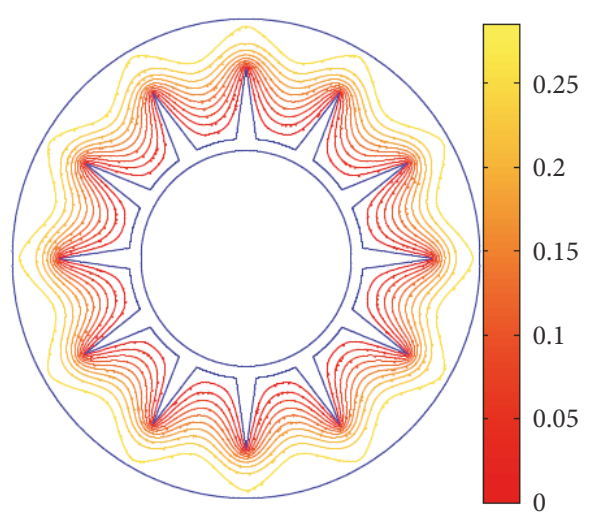

(x) $\Omega=1000$

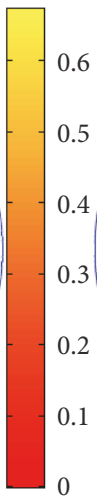

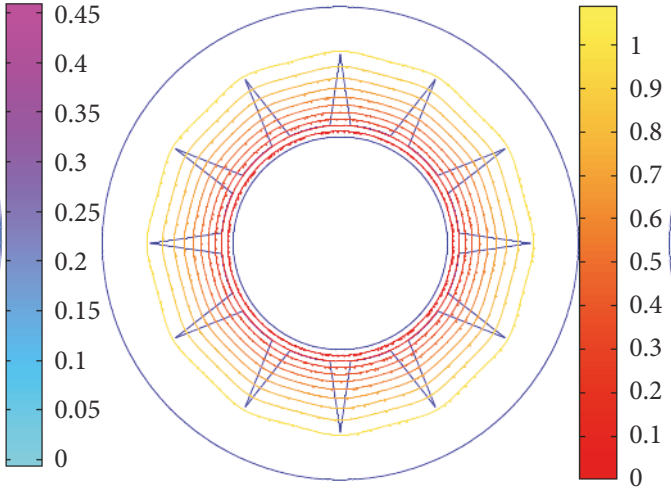

(ii) $\Omega=1$

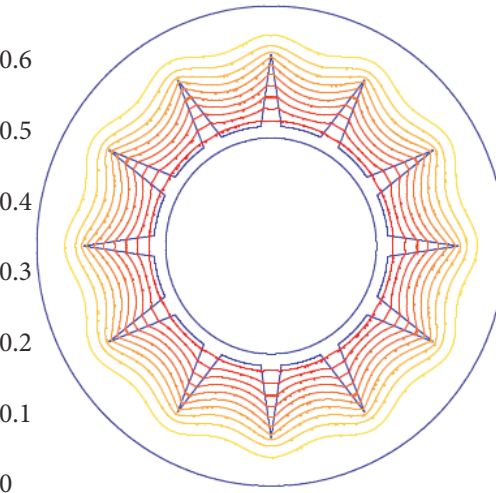

(v) $\Omega=10$

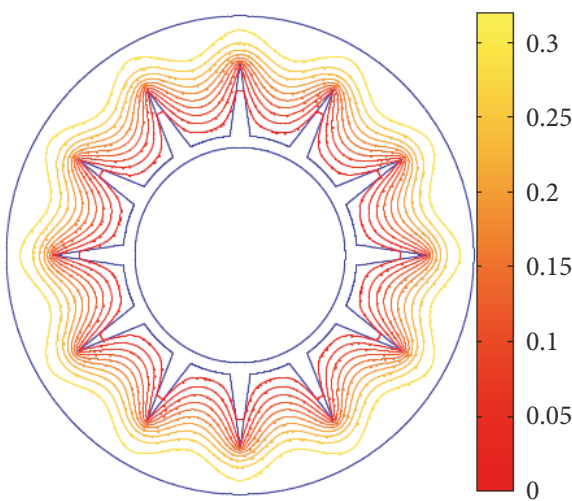

(viii) $\Omega=100$

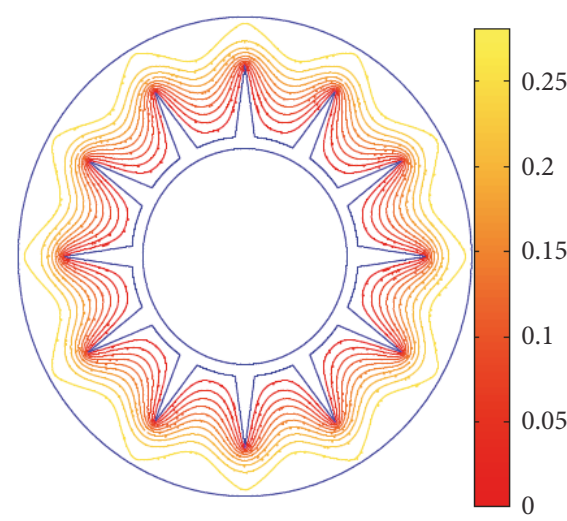

(xi) $\Omega=5000$

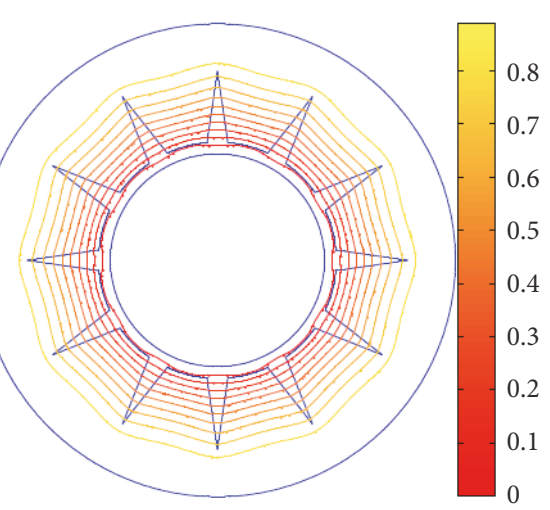

(iii) $\Omega=2$

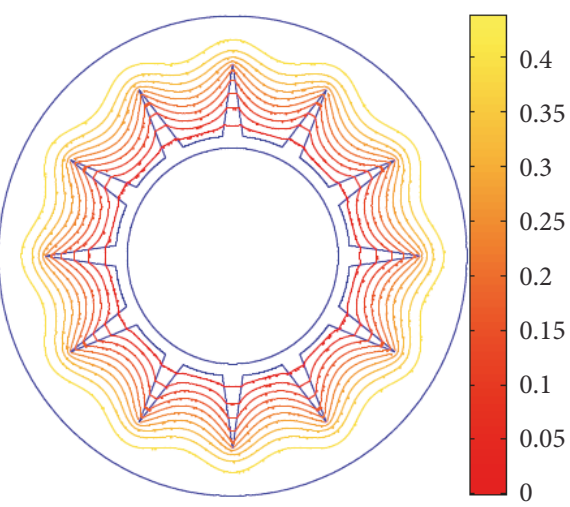

(vi) $\Omega=20$

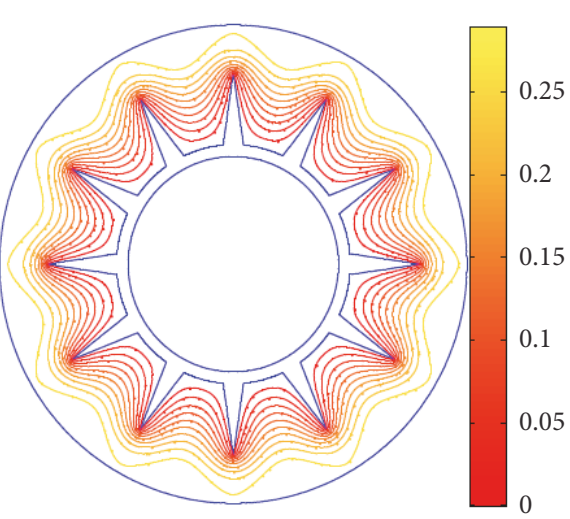

(ix) $\Omega=500$

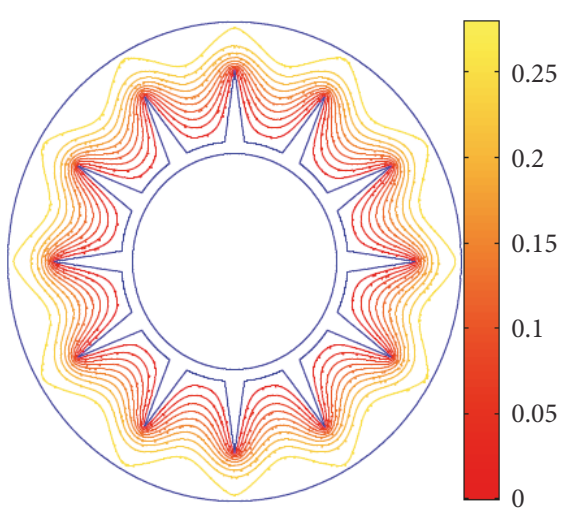

(xii) $\Omega=\infty$

(a) Velocity contours and isotherms at $\widehat{R}=0.5, \beta=5^{\circ}, \delta=0.05, M=12, H^{*}=0.6$, and $1 \leq \Omega \leq \infty$. (i) Velocity contours, (ii) $\Omega=1$ (iii) $\Omega=2$, (iv) $\Omega=5$, (v) $\Omega=10$, (vi) $\Omega=20$, (vii) $\Omega=50$, (viii) $\Omega=100$, (ix) $\Omega=500$, (x) $\Omega=1000$, (xi) $\Omega=5000$, and (xii) $\Omega=\infty$ 


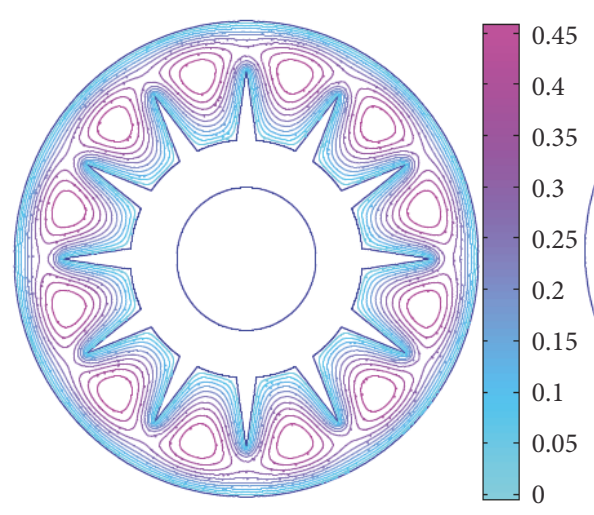

(i)

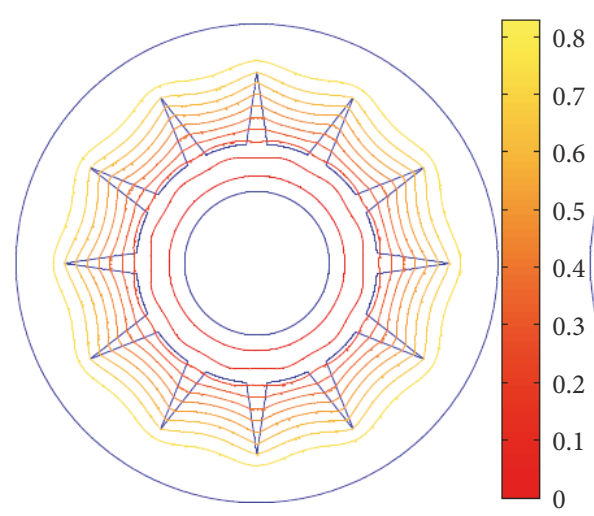

(iv) $\Omega=5$

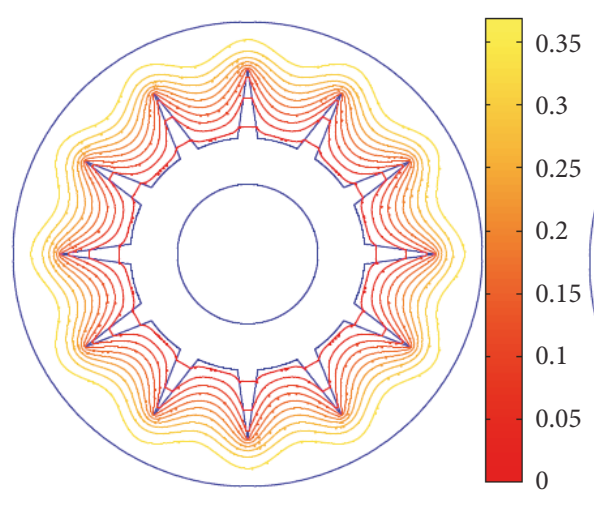

(vii) $\Omega=50$

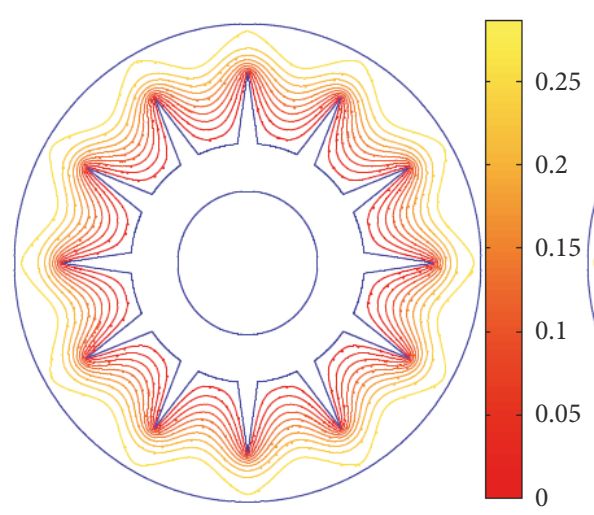

(x) $\Omega=1000$

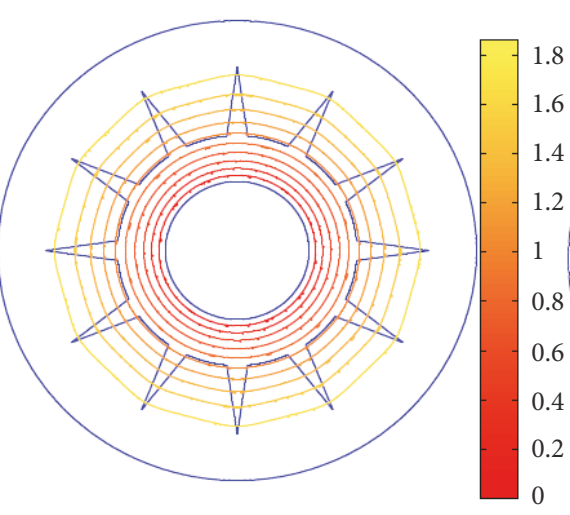

(ii) $\Omega=1$

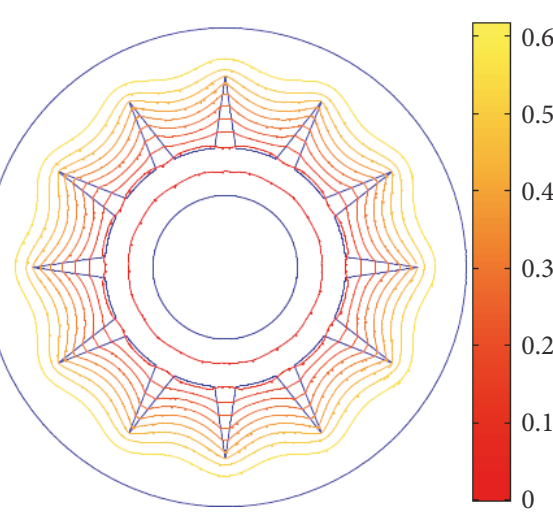

(v) $\Omega=10$

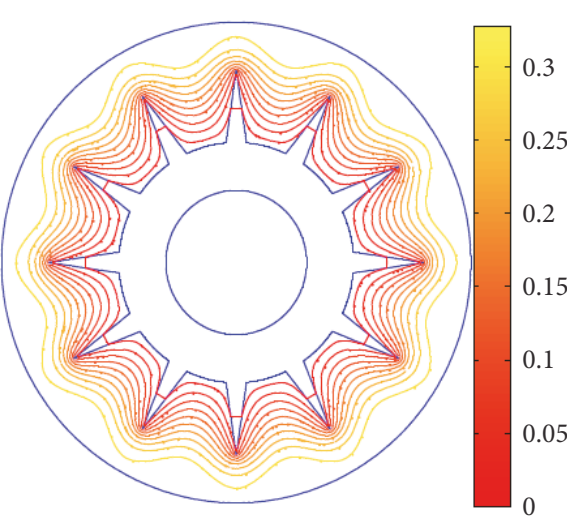

(viii) $\Omega=100$

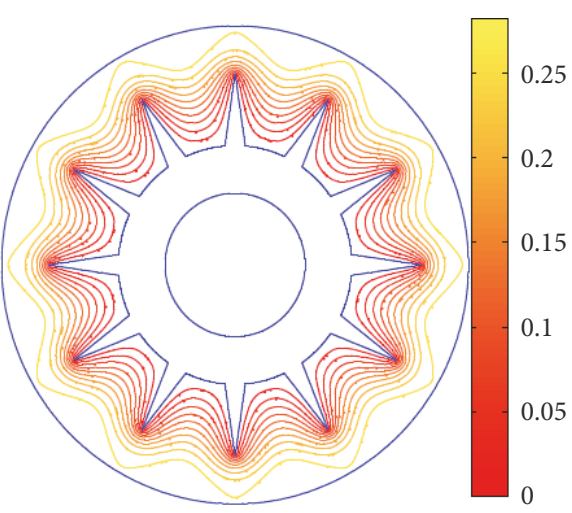

(xi) $\Omega=5000$

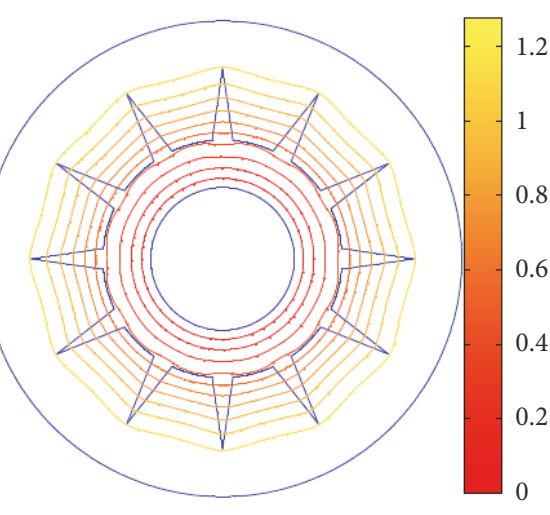

(iii) $\Omega=2$

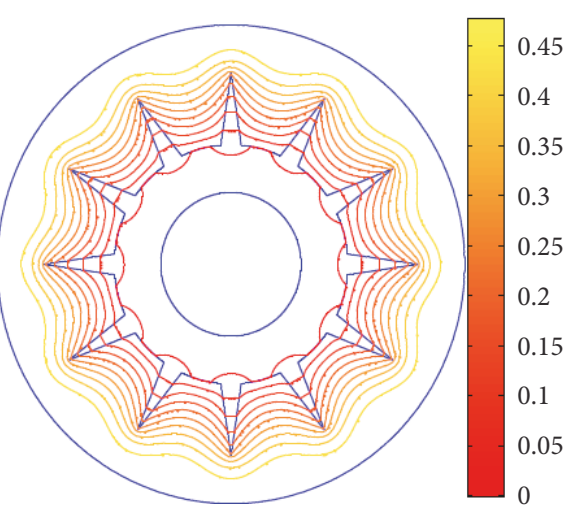

(vi) $\Omega=20$

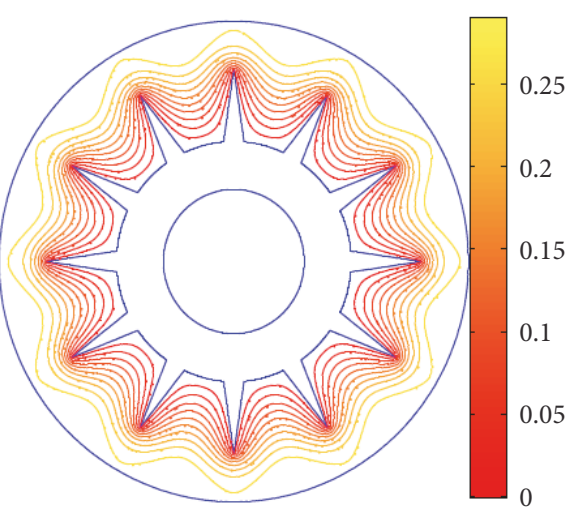

(ix) $\Omega=500$

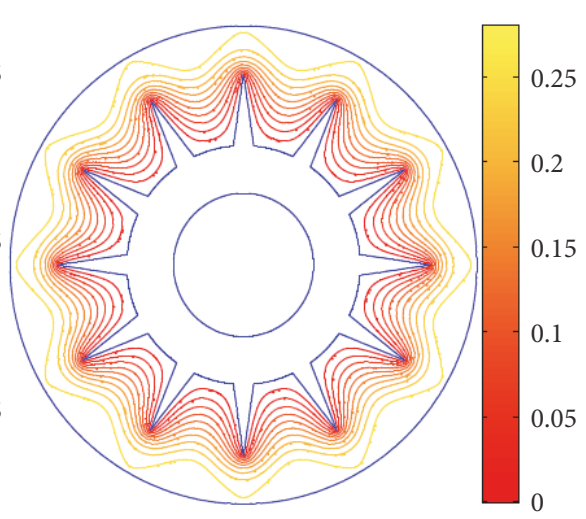

(xii) $\Omega=\infty$

(b) Velocity contours and isotherms at $\widehat{R}=0.5, \beta=5^{\circ}, \delta=0.2, M=12, H^{*}=0.6$, and $1 \leq \Omega \leq \infty$. (i) Velocity contours (ii) $\Omega=1$ (iii) $\Omega=2$, (iv) $\Omega=5$, (v) $\Omega=10$, (vi) $\Omega=20$, (vii) $\Omega=50$, (viii) $\Omega=100$, (ix) $\Omega=500$, (x) $\Omega=1000$, (xi) $\Omega=5000$, (xii) $\Omega=\infty$ 


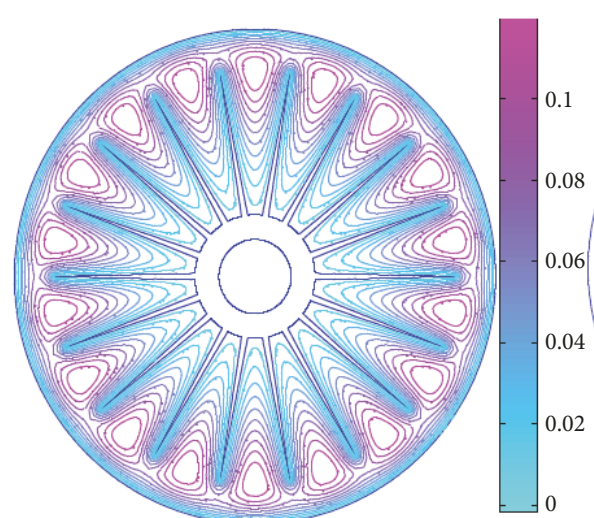

(i)

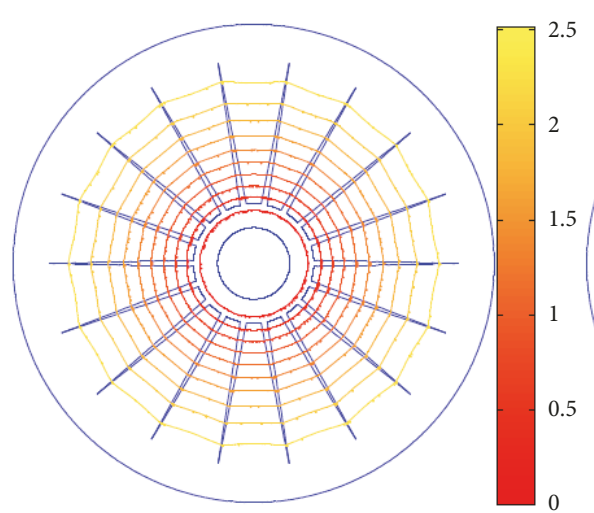

(iv) $\Omega=5$

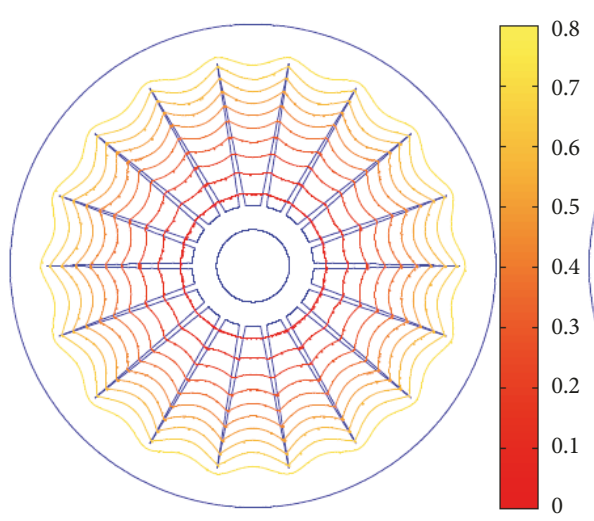

(vii) $\Omega=50$

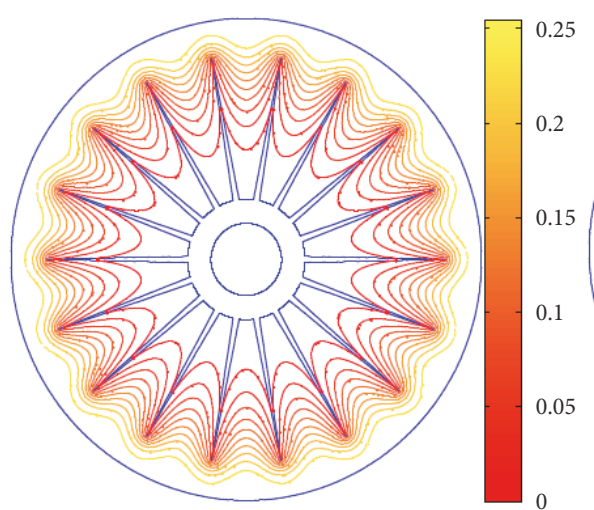

(x) $\Omega=1000$

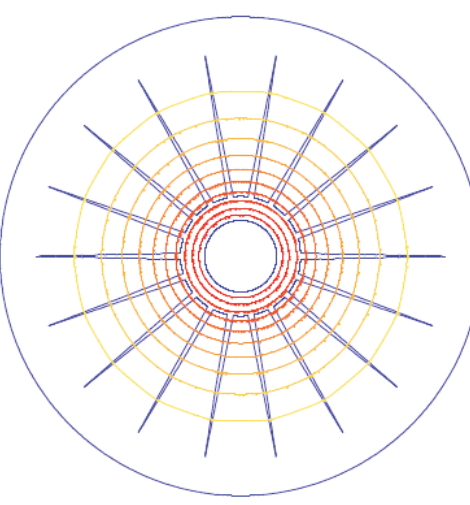

(ii) $\Omega=1$

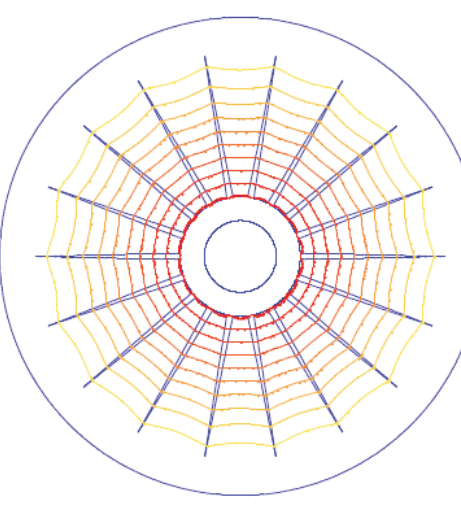

(v) $\Omega=10$

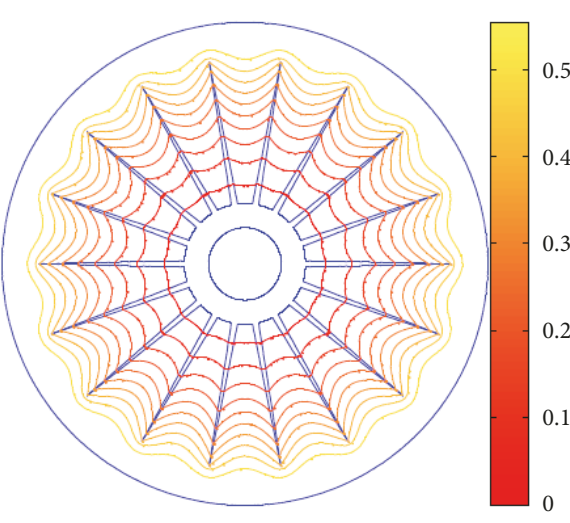

(viii) $\Omega=100$

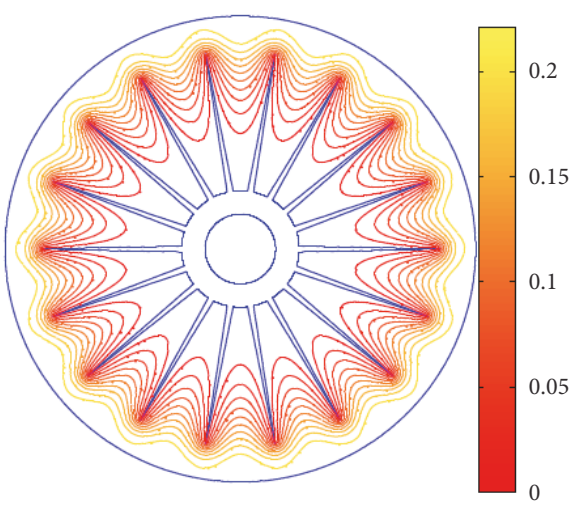

(xi) $\Omega=5000$

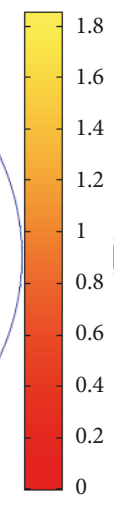

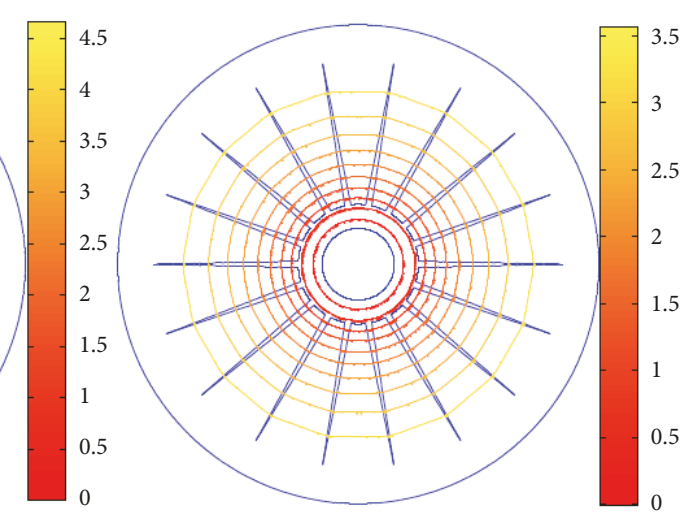

(iii) $\Omega=2$

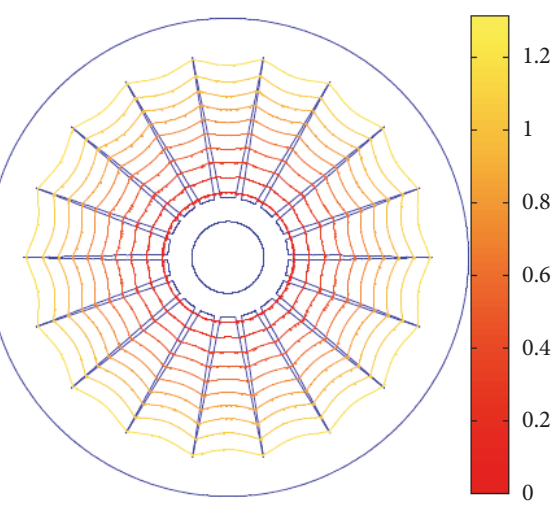

(vi) $\Omega=20$

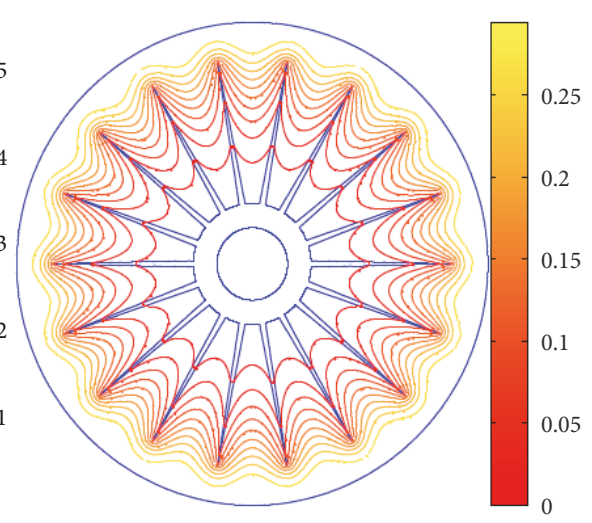

(ix) $\Omega=500$

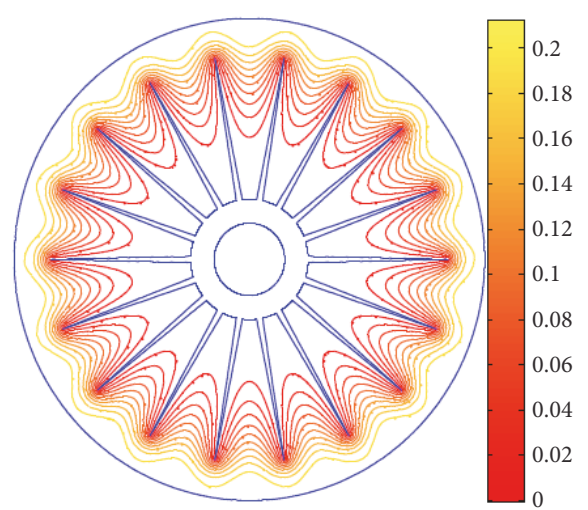

(xii) $\Omega=\infty$

(c) Velocity contours and isotherms at $\widehat{R}=0.25, \beta=3^{\circ}, \delta=0.1, M=18, H^{*}=0.8$ and $1 \leq \Omega \leq \infty$. (i) Velocity contours, (ii) $\Omega=1$ (iii) $\Omega=2$, (iv) $\Omega=5$, (v) $\Omega=10$, (vi) $\Omega=20$, (vii) $\Omega=50$, (viii) $\Omega=100$, (ix) $\Omega=500$, (x) $\Omega=1000$, (xi) $\Omega=5000$, and (xii) $\Omega=\infty$ 


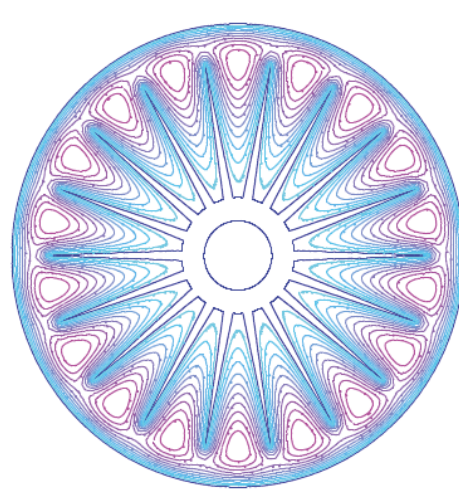

(i)

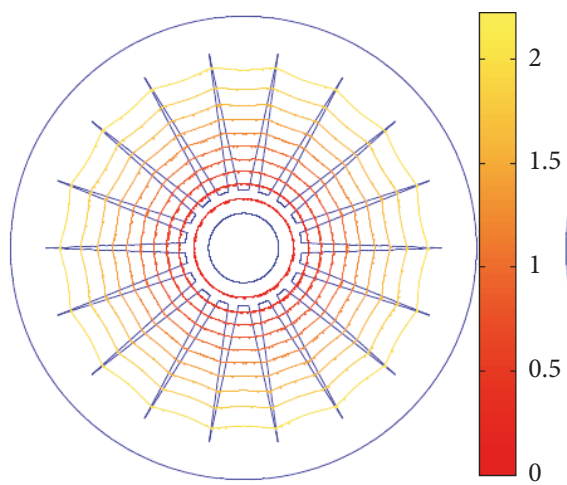

(iv) $\Omega=5$

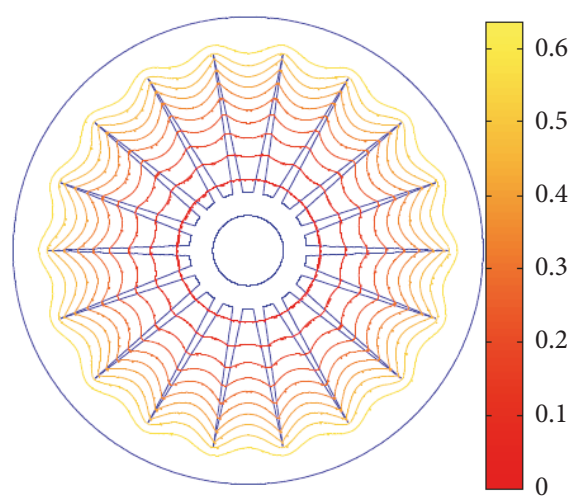

(vii) $\Omega=50$

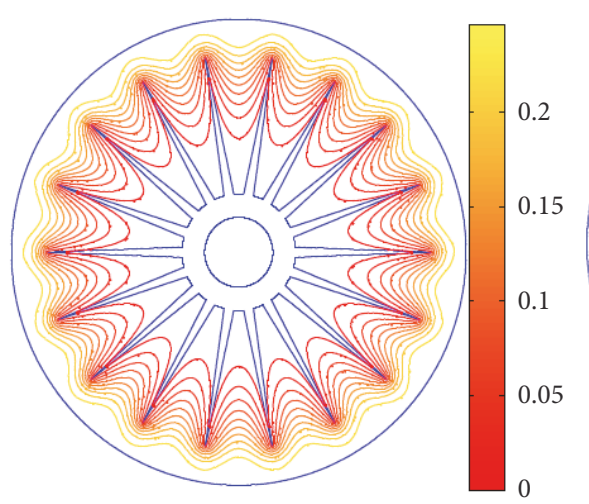

(x) $\Omega=1000$

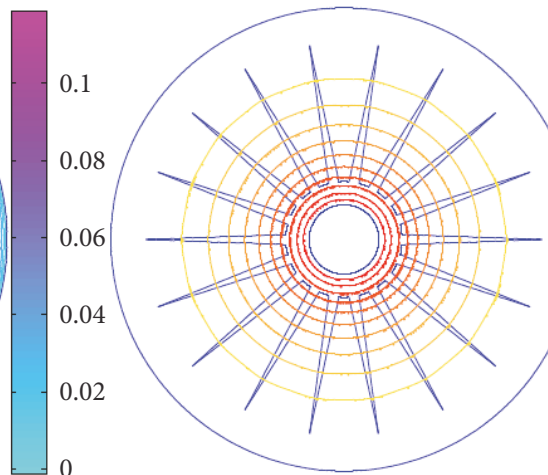

(ii) $\Omega=1$

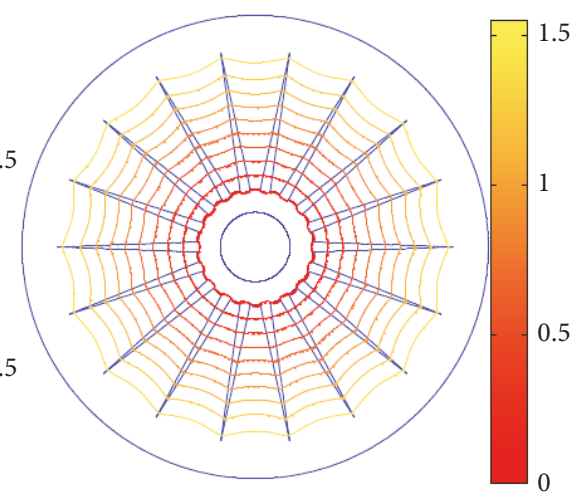

(v) $\Omega=10$

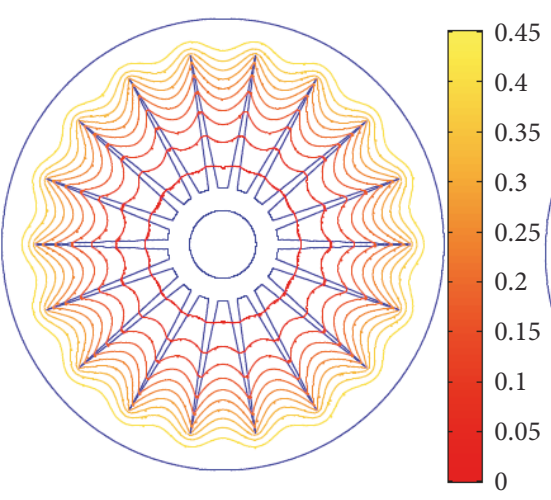

(viii) $\Omega=100$

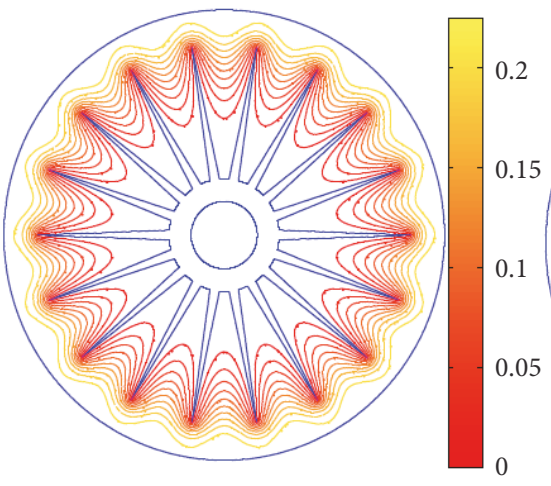

(xi) $\Omega=5000$
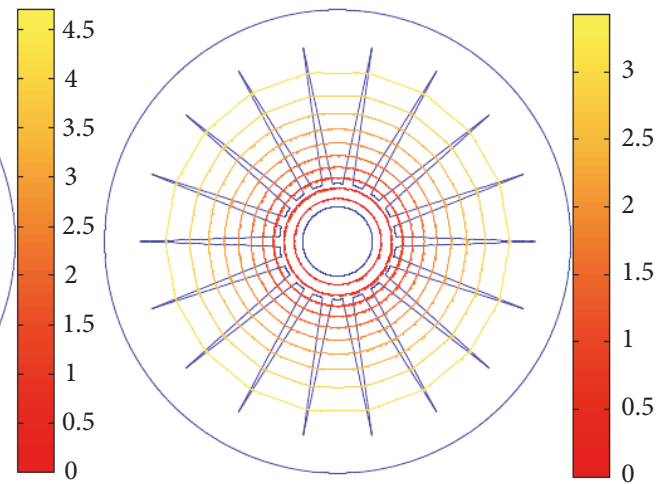

(iii) $\Omega=2$

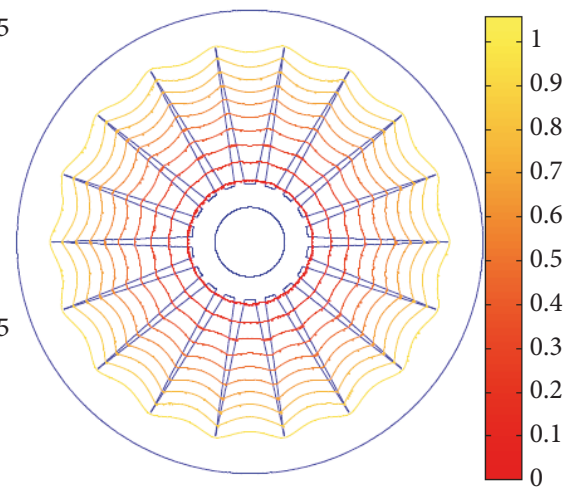

(vi) $\Omega=20$

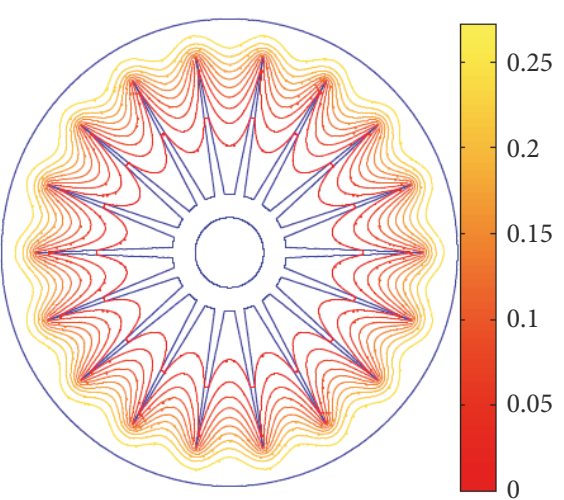

(ix) $\Omega=500$

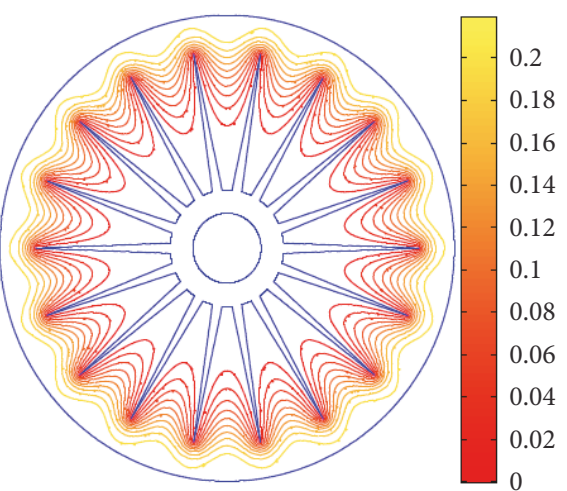

(xii) $\Omega=\infty$

(d) Velocity contours and isotherms at $\widehat{R}=0.25, \beta=5^{\circ}, \delta=0.1, M=18, H^{*}=0.8$, and $1 \leq \Omega \leq \infty$. (i) Velocity contours, (ii) $\Omega=1$ (iii) $\Omega=2$, (iv) $\Omega=5$, (v) $\Omega=10$, (vi) $\Omega=20$, (vii) $\Omega=50$, (viii) $\Omega=100$, (ix) $\Omega=500$, (x) $\Omega=1000$, (xi) $\Omega=5000$, and (xii) $\Omega=\infty$ 


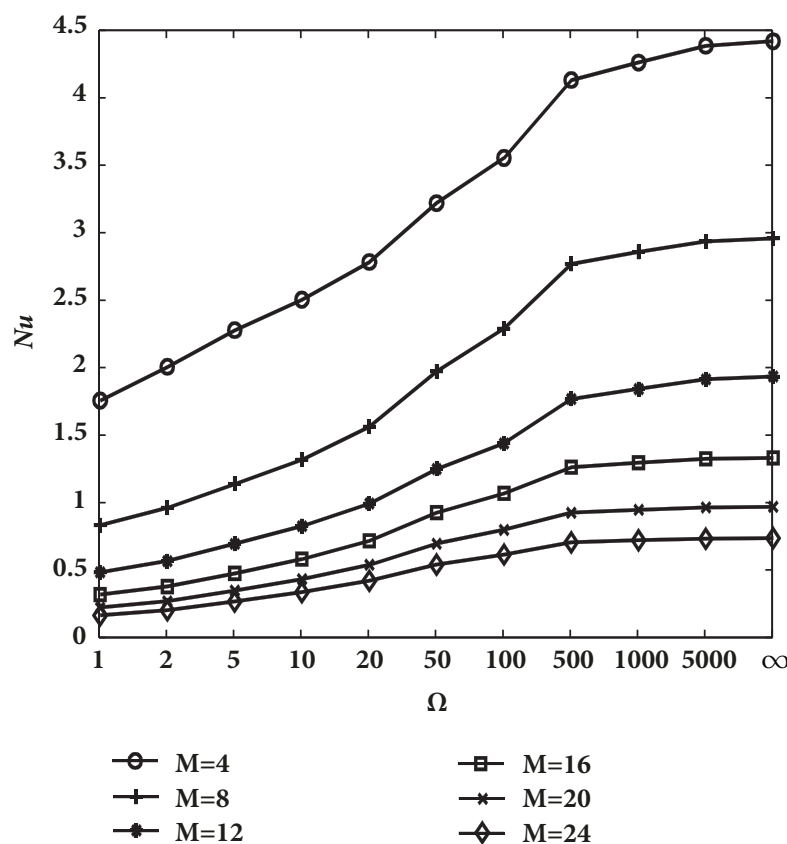

(a) $H^{*}=0.4, \delta=0.05$

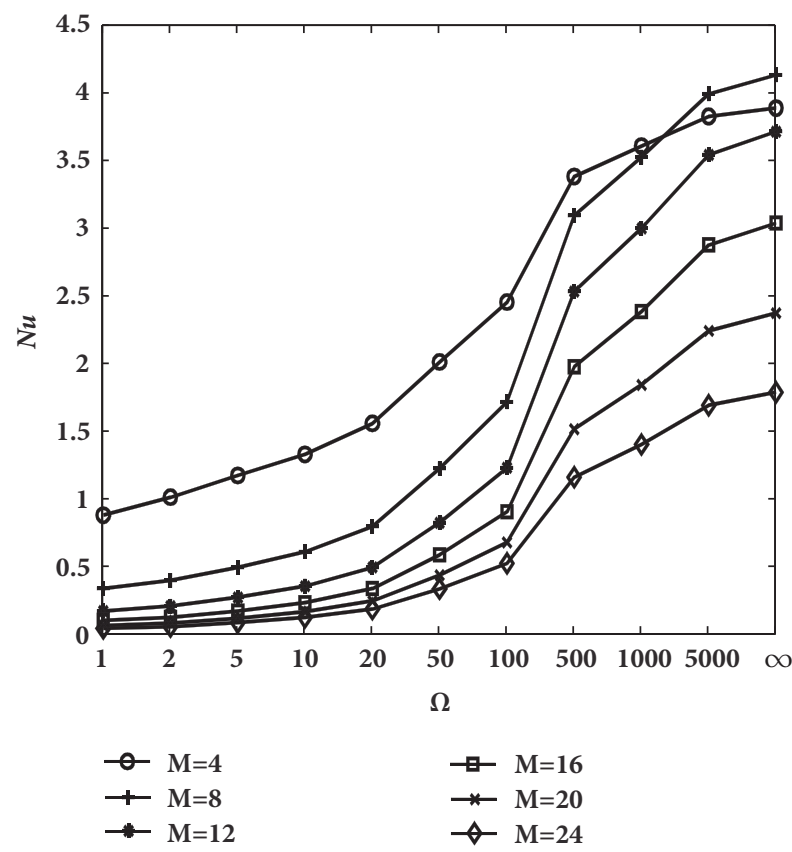

(c) $H^{*}=0.8, \delta=0.05$

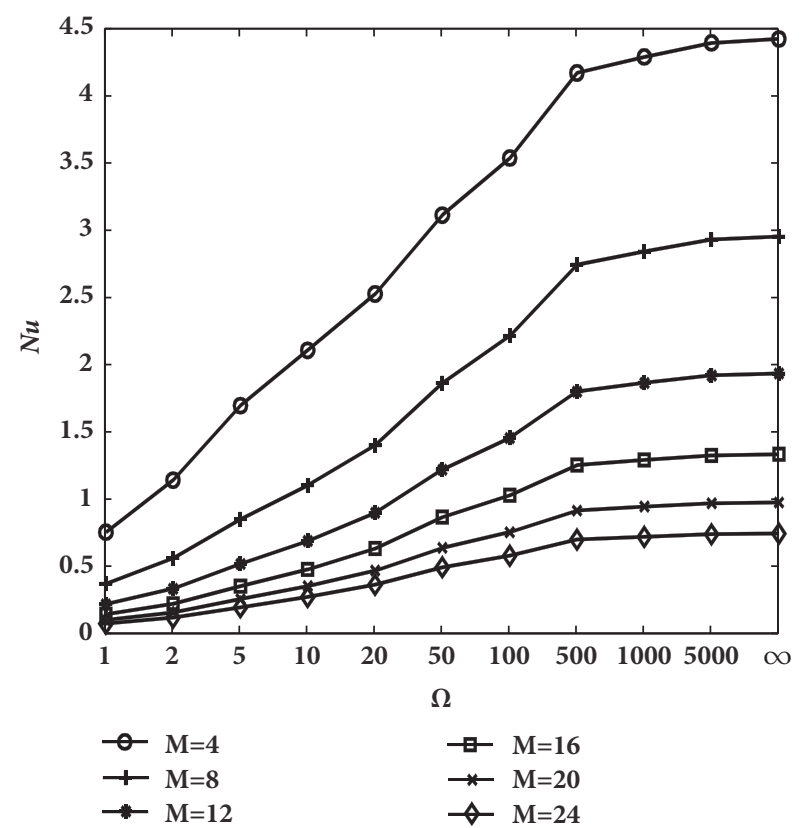

(b) $H^{*}=0.4, \delta=0.2$

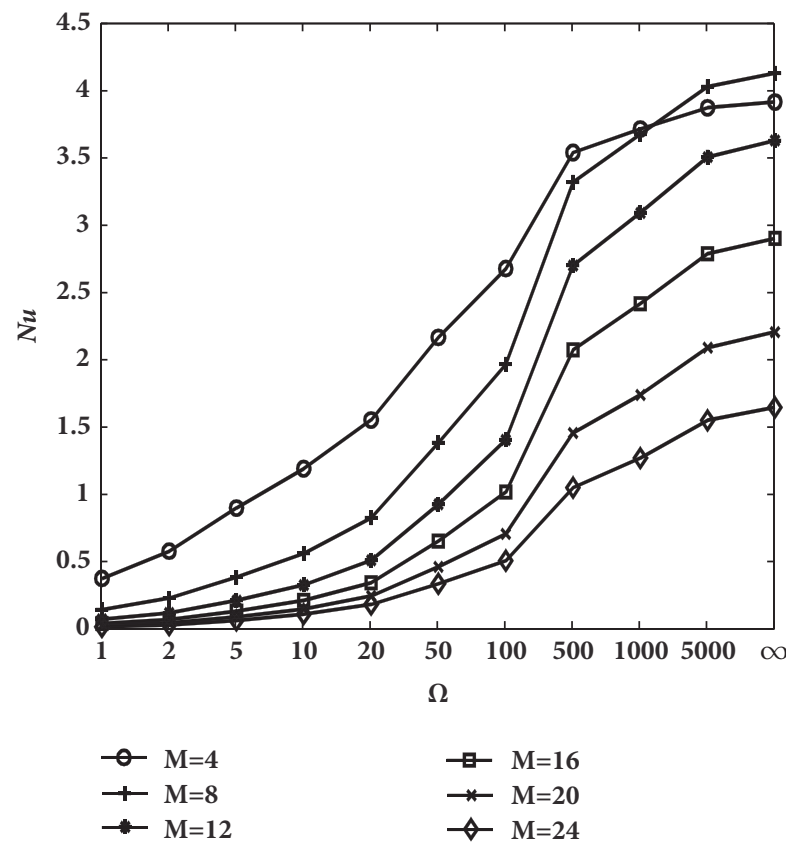

(d) $H^{*}=0.8, \delta=0.2$

FigURE $4: \overline{N u}$ is plotted against the ratio of conductivities $\Omega$ at $\widehat{R}=0.25, \beta=3^{\circ}$.

Triangular fin height also assists the inner pipe wall thickness in its behavior described earlier.

Figure 5 represents the graph between $\overline{N u}$ and $\Omega$ at $\widehat{R}=$ $0.6, \beta=3^{\circ}, H^{*}=0.4$ and $0.8, \delta=0.05$ and 0.2 , and $4 \leq$ $M \leq 24$. Most of previous comments are also applicable here. On comparing Figures 4 and 5, the effect of increasing the value of $\widehat{R}$ on the Nusselt number may be seen. It may be noted from this comparison that, due to the increase of the ratio of radii, intercurves spacing for triangular fins of $40 \%$ height and nonuniform behavior of the Nusselt number with the number of triangular fins for various values of the conductivity ratios tend to become uniform. However, for the case of larger triangular fins with $80 \%$ height and for large values of $\Omega$ an increase in the values of $\widehat{R}$ makes the trend 

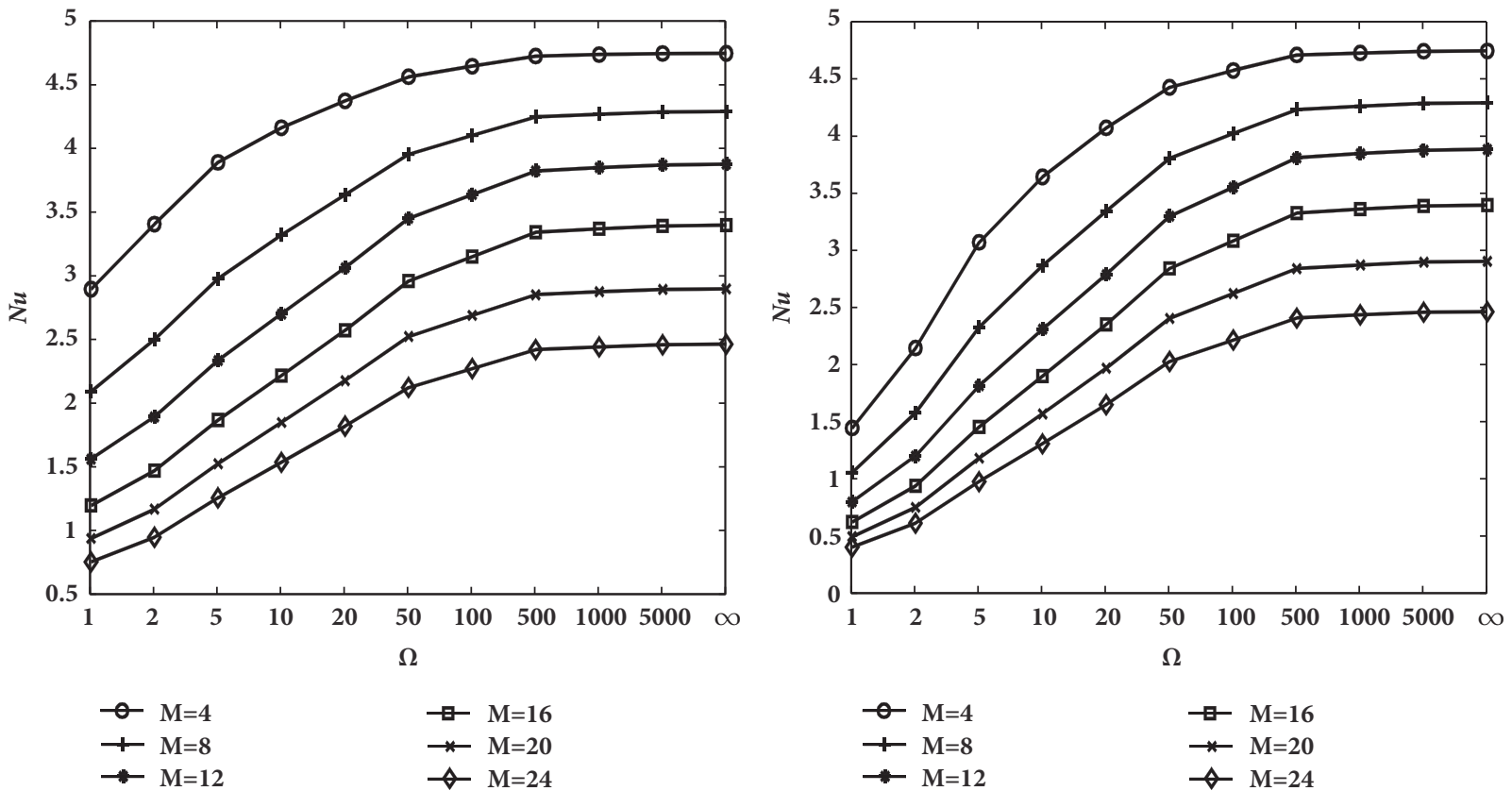

(a) $H^{*}=0.4, \delta=0.05$
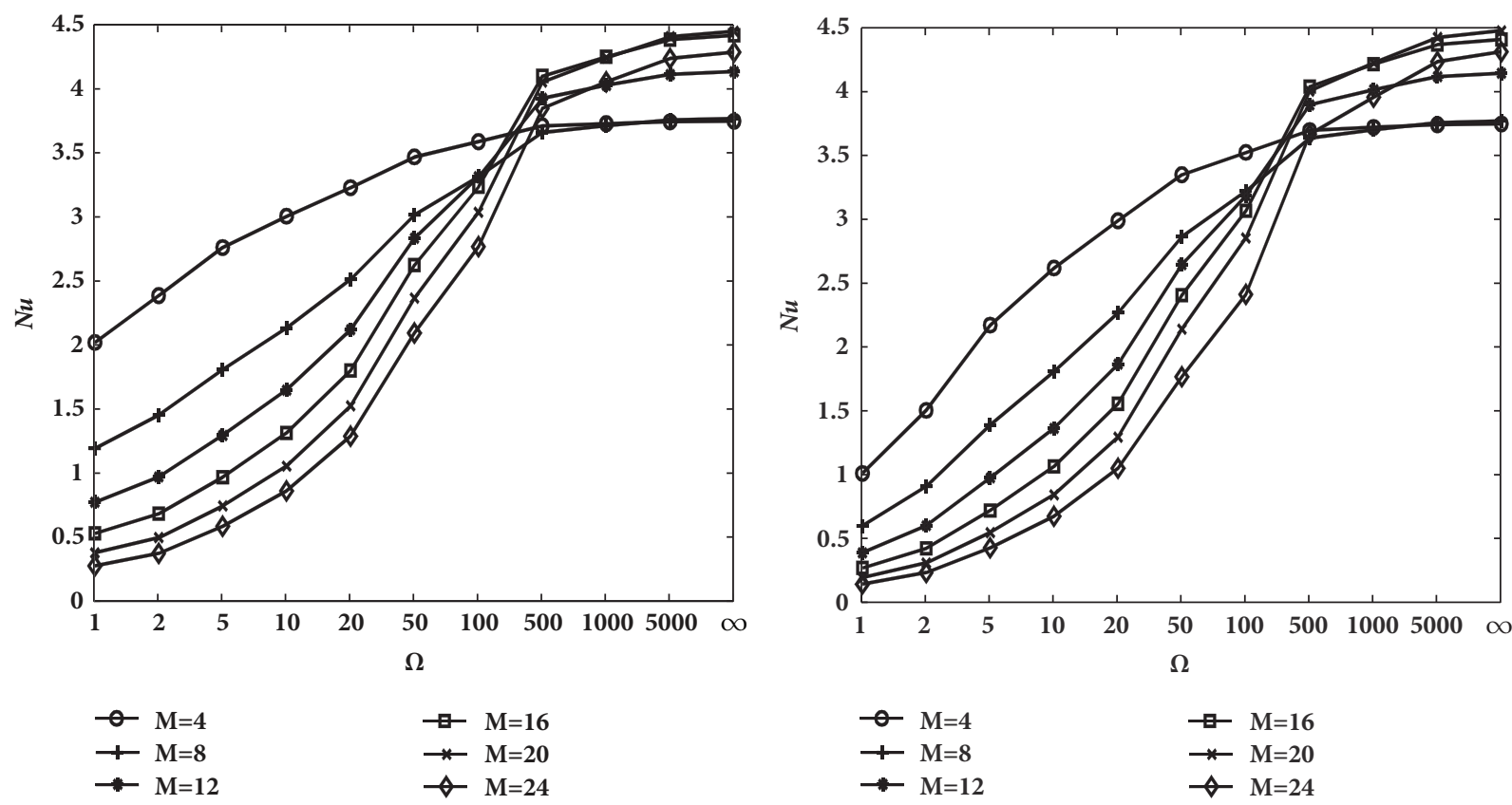

(c) $H^{*}=0.8, \delta=0.05$

(d) $H^{*}=0.8, \delta=0.2$

Figure 5: A graph of $\overline{N u}$ and $\Omega$ at $\widehat{R}=0.6, \beta=3^{\circ}$.

of $\overline{N u}$ curves more complex. It may be seen that at these values of $\Omega$ the number of intersections of different $\overline{N u}$ curves increases with $\widehat{R}$.

A graph is sketched between $\overline{N u}$ and $\Omega$ in Figure 6 for $\widehat{R}=$ $0.6, \beta=5^{\circ}, H^{*}=0.4$ and $0.8, \delta=0.05$ and 0.2 , and $4 \leq M \leq 24$. Most of the comments made for Figure 5 are also applicable here. The comparison of Figures 5 and 6 states that, for larger value of $\widehat{R}$, the influence of the triangular fin half angle on
$\overline{\mathrm{Nu}}$ becomes more substantial particularly for larger values of triangular fin height and the conductivity ratios.

The influence of increasing triangular fin height on the present problem may be seen in Figure 7, where $\overline{N u}$ is plotted against $\Omega$ at $\widehat{R}=0.25, \beta=3^{\circ}, 0.2 \leq H^{*} \leq 0.8, \delta=0.05$, and $4 \leq M \leq 16$. It may be observed from this figure that, with increase in the triangular fin height, $\overline{N u}$ decrease particularly for smaller number of fins and for all $\Omega$ while 

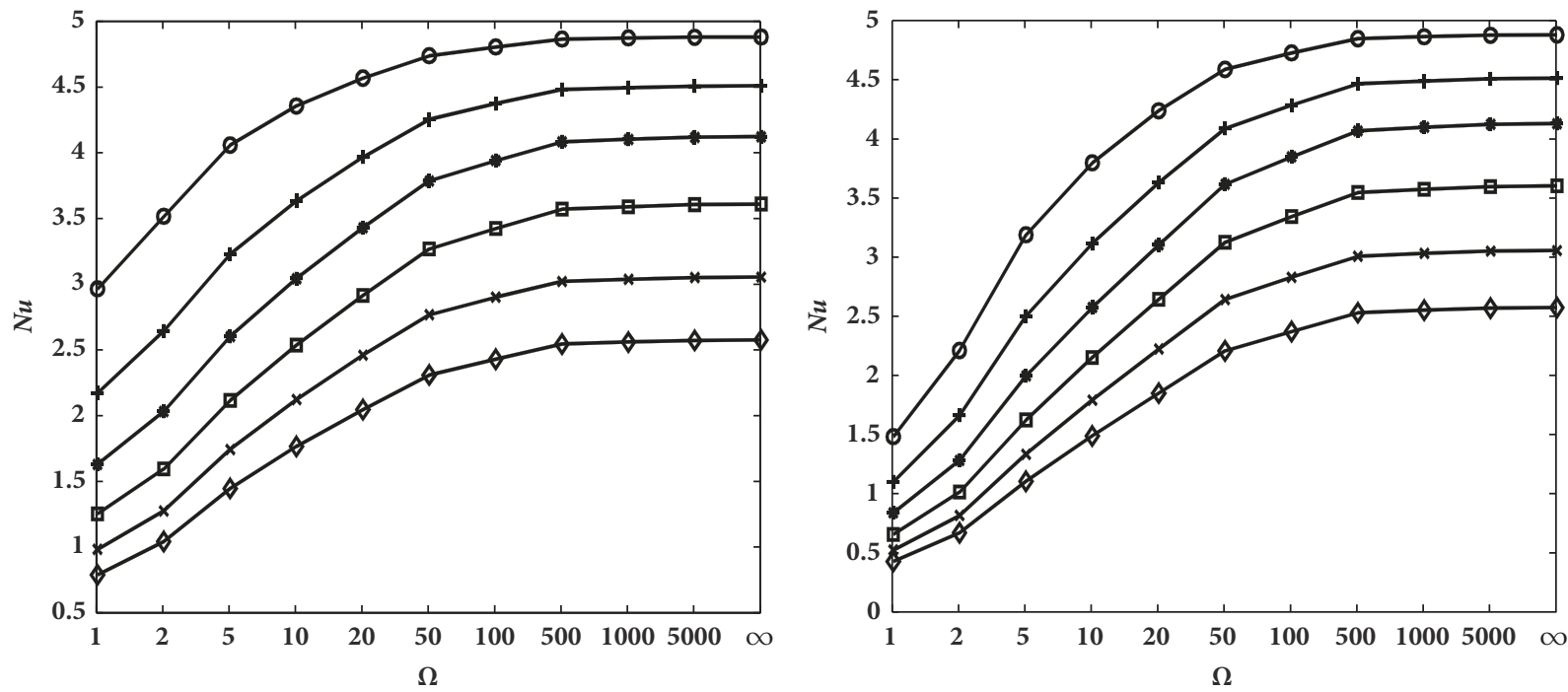

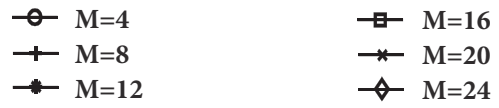

(a) $H^{*}=0.4, \delta=0.05$
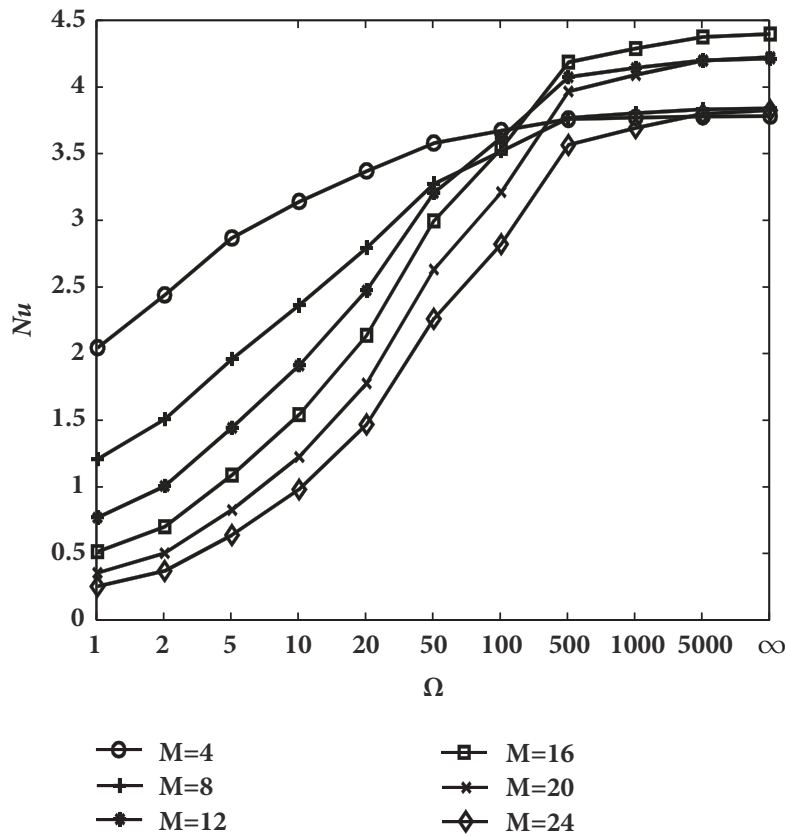

(c) $H^{*}=0.8, \delta=0.05$

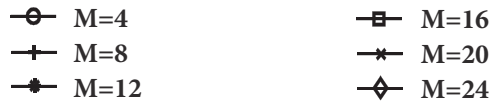

(b) $H^{*}=0.4, \delta=0.2$
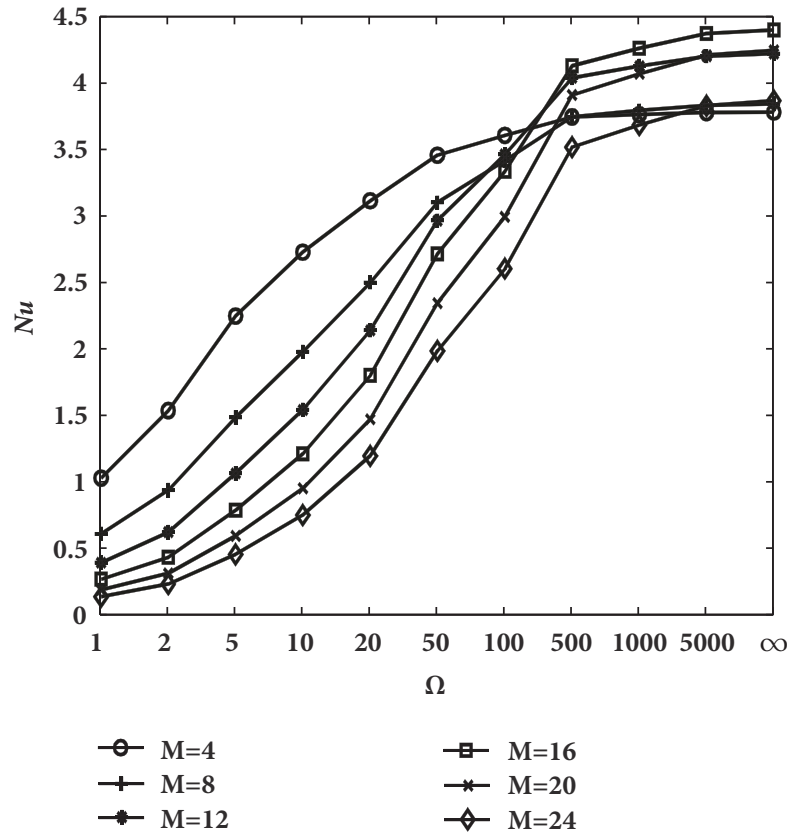

(d) $H^{*}=0.8, \delta=0.2$

Figure 6: A graph between $\overline{N u}$ and $\Omega$ at $\widehat{R}=0.6, \beta=5^{\circ}$.

for higher number of fins and $\Omega$ the triangular fins of larger height become relatively more active. It may be further noted that, for $\Omega \geq 500$, the trend of $\overline{N u}$ curves becomes almost horizontal when $H^{*} \leq 0.4$ for other specified values of $\widehat{R}, \beta$, $\delta$, and $M$. This trend of curves shows that the assumption of negligible thermal resistance [15] may be safely applied when the conductivity ratio for the materials of inner pipe wall-fin assembly is less than 500 and the configurations of triangular FDP with fin height are not more than $40 \%$ of the annulus of FDP. However, this assumption may not be employed for larger triangular fin heights because of substantial influence in $\overline{N u}$. It may be concluded that for choice of the range of triangular fin heights to consider the assumption of infinite conductivity strongly depends on the geometric parameters especially the number of fins.

For the validation of this assumption which is considered here, Table 1 represents the comparison with [15] in quantitative form. This table presents percentage overestimates in the 


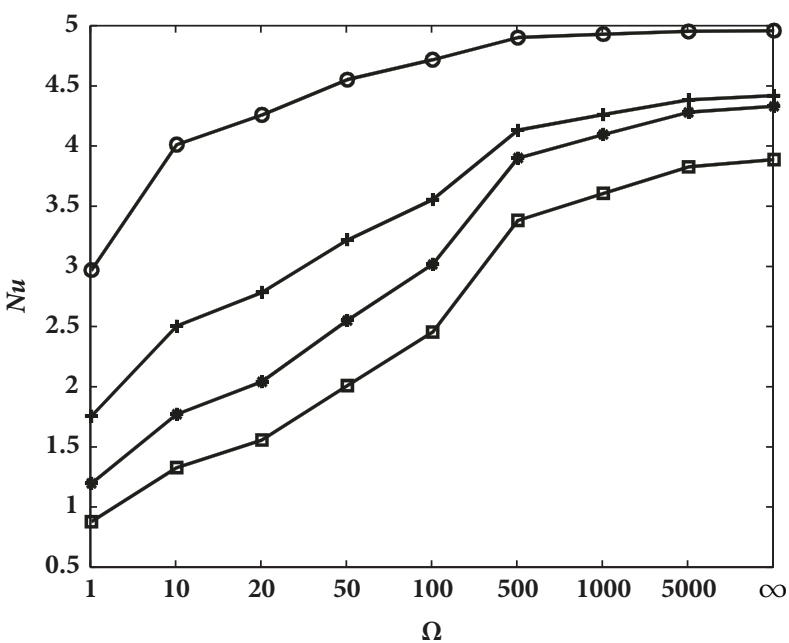

$$
\begin{aligned}
-0 & H^{*}=0.2 \\
+- & H^{*}=0.4
\end{aligned}
$$

(a) $M=4$

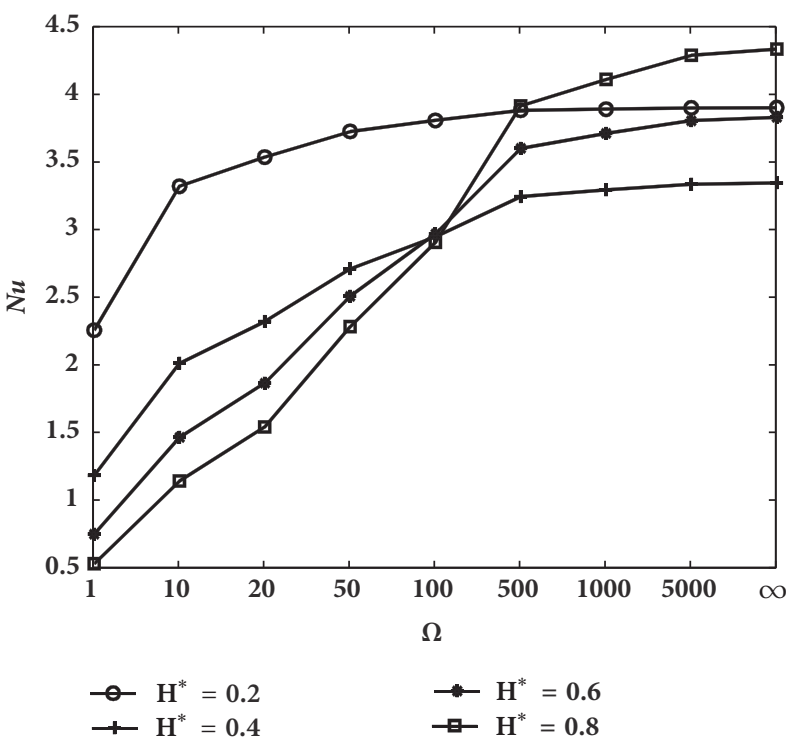

(c) $M=12$

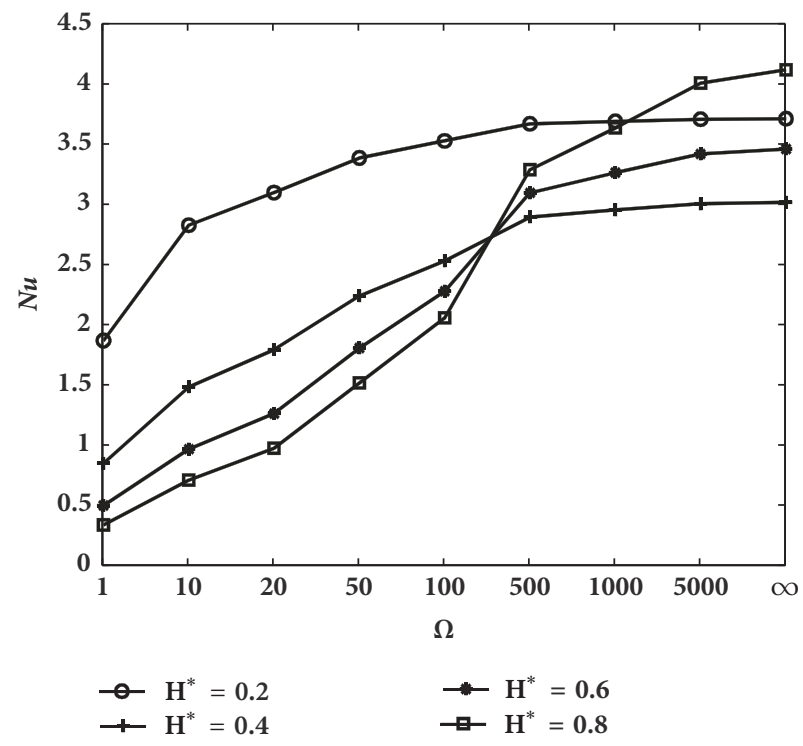

(b) $M=8$

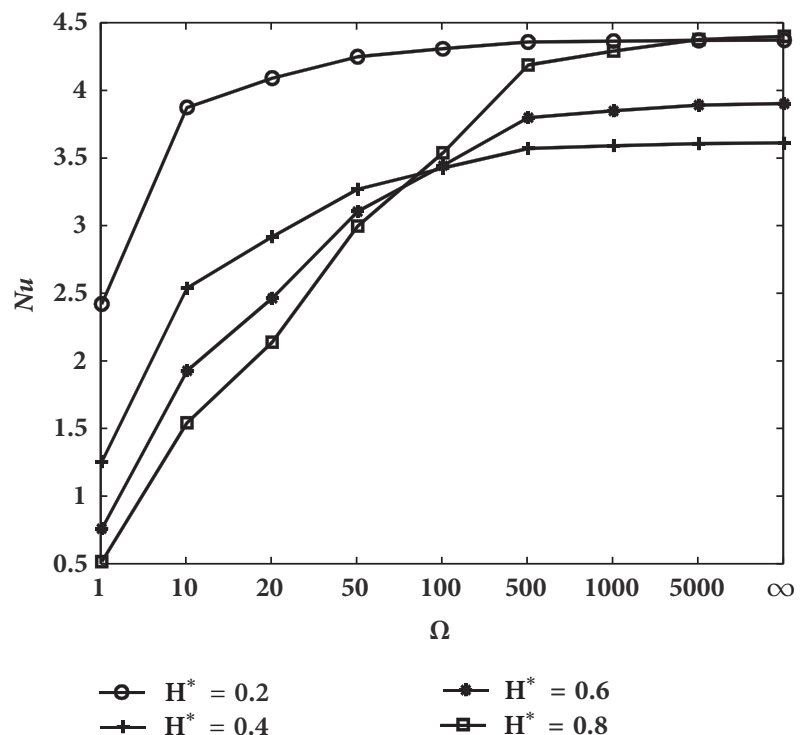

(d) $M=16$

Figure 7: $\overline{N u}$ is plotted against $\Omega$ at $\widehat{R}=0.25, \beta=3^{\circ}, \delta=0.05$, and $4 \leq M \leq 16$.

values of the Nusselt number for $\widehat{R}=0.5, \beta=3^{\circ}, \delta=0.1$, and $1 \leq \Omega \leq 5000$. These overestimates in $\overline{\mathrm{Nu}}$ are due to employing this assumption when finite conductivity ratio $(1 \leq \Omega \leq 5000)$ is considered.

If up to $5 \%$ overestimate in $\overline{N u}$ is tolerable, then it may be noted from Table 1 that the assumption of negligible thermal resistance of the material of inner pipe wall-fin is not valid for any choice of triangular fin height and the number of fins for $\Omega<50$.

However, the choice of $\Omega$ for the validity of this assumption varies with increase in triangular fin height and number of fins. It may be noted that, for the case of $20 \%$ triangular fin height, it is valid for $\Omega \geq 100$ and all numbers of triangular fins; for $40 \%$ triangular fin height, it is true for $\Omega \geq 500$ and all number of triangular fins; for $60 \%$ triangular fin height it is valid for $\Omega \geq 500$ for $M \leq 8$ and $\Omega \geq 1000$ for $M \geq 12$; for $80 \%$ triangular fin height it is true for $\Omega \geq 500$ for $M=4$, $\Omega \geq 1000$ for $M=8$, and $\Omega \geq 5000$ for $M \geq 12$.

A graph of $\overline{N u}$ is sketched against $M$ for $0.05 \leq \delta \leq$ $0.2,0.2 \leq H^{*} \leq 0.8,4 \leq M \leq 24,1 \leq \Omega \leq 5000$, and $\beta=3^{\circ}$ in Figure 8 , for the validity of the assumption of negligible/zero inner pipe wall thickness considered in [15]. From this figure, one may conclude that, for small values of the conductivity ratios, number of triangular fins, and fin height, the Nusselt number significantly depends on the thickness of the inner pipe wall. As $\Omega$ gradually increase its 

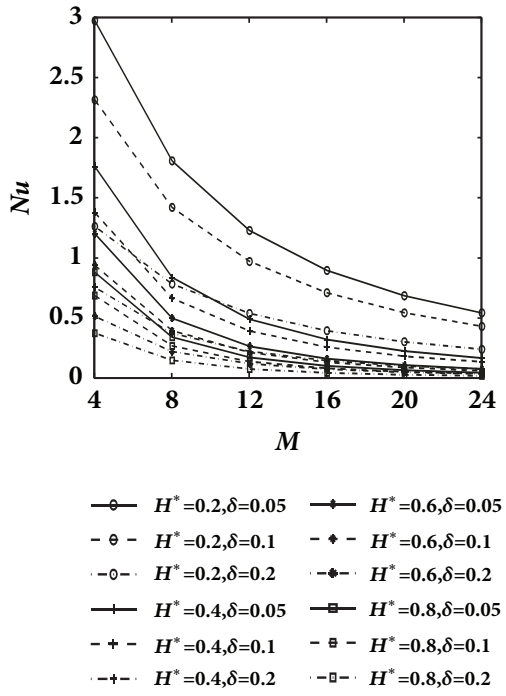

(a) $\Omega=1$

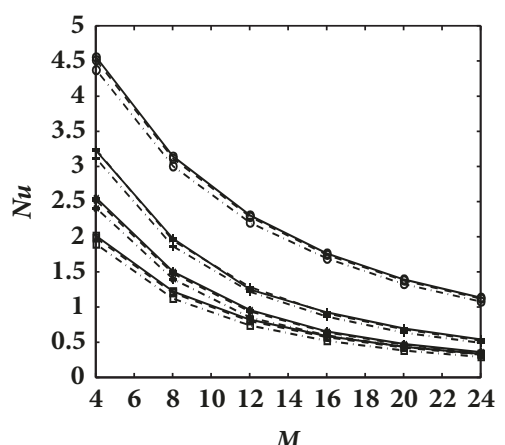

M

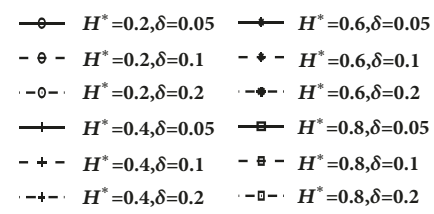

(d) $\Omega=50$
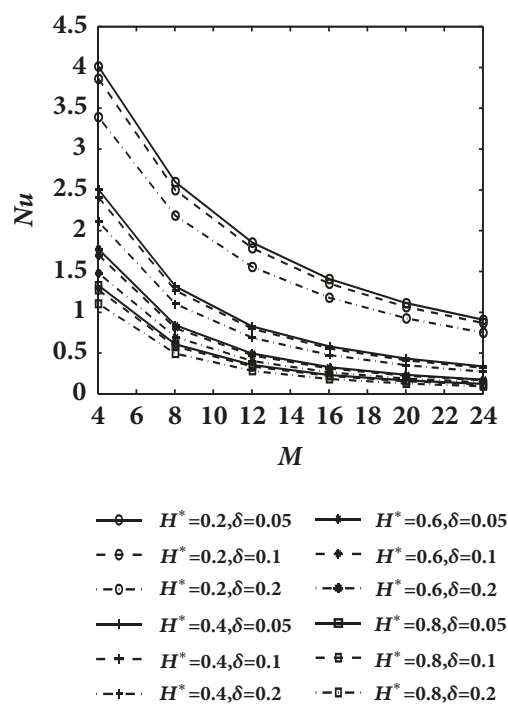

(b) $\Omega=10$

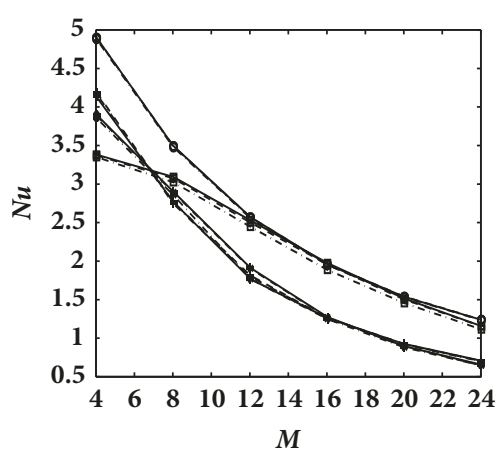

$\rightarrow H^{*}=0.2, \delta=0.05 \rightarrow-H^{*}=0.6, \delta=0.05$

$-\theta-H^{*}=0.2, \delta=0.1 \quad-H^{*}=0.6, \delta=0.1$

- 0 - $H^{*}=0.2, \delta=0.2 \quad \cdots-H^{*}=0.6, \delta=0.2$

一 $H^{*}=0.4, \delta=0.05 \rightarrow H^{*}=0.8, \delta=0.05$

- + - $H^{*}=0.4, \delta=0.1 \quad-\quad-H^{*}=0.8, \delta=0.1$

- -+- $H^{*}=0.4, \delta=0.2 \quad-\square-H^{*}=0.8, \delta=0.2$

(e) $\Omega=500$
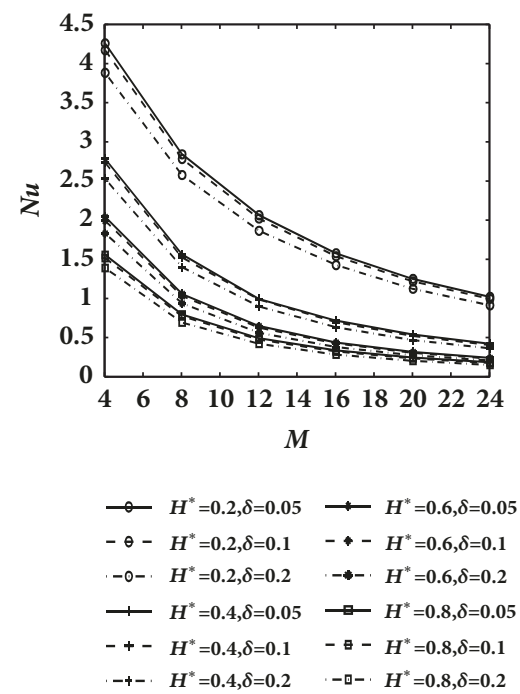

(c) $\Omega=20$
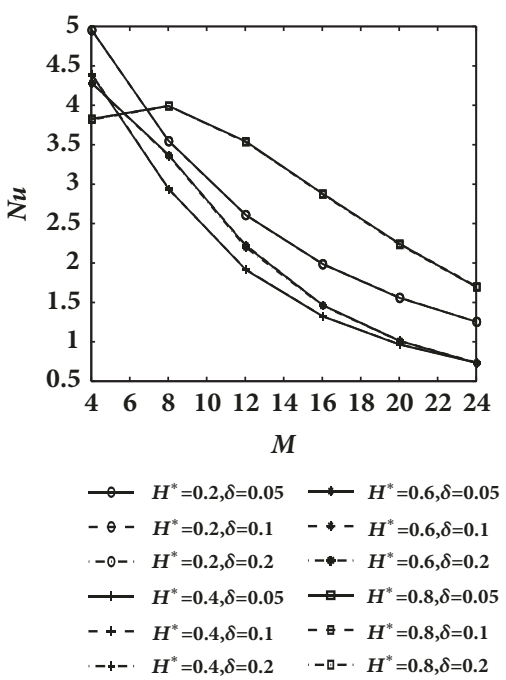

(f) $\Omega=5000$

FIGURE 8: $\overline{N u}$ is plotted against $M$ for $0.05 \leq \delta \leq 0.2,0.2 \leq H^{*} \leq 0.8,4 \leq M \leq 241 \leq \Omega \leq 5000$, and $\beta=3^{\circ}$.

value, the $\overline{N u}$ curves representing different values of $\delta$ get closer and closer showing negligible influence of inner pipe wall thickness for any values of $M$ and $H^{*}$. The present results suggest that the assumption of negligible/zero inner pipe wall thickness can safely be employed for $\Omega \geq 500$.

Now, the Nusselt number based on the equivalent diameter $\overline{N u}_{e}$ is plotted against $\Omega$ at $\widehat{R}=0.5, \beta=3^{\circ}$, and $\delta=0.05$ in Figure 9. It may be noted that $\overline{N u}_{e}$ curves present increasing trend with increase in $\Omega$, for all choices of triangular fin height and number of fins. It can be seen that, for lower values of $\Omega$, there is no significant change in $\overline{N u}_{e}$ curves for specified values of $H^{*}$ and $M$ while $\overline{N u}_{e}$ show significant increase in the values for higher $\Omega$. For lower values of $\Omega$ and for any fixed $H^{*}$, the effect of increasing the number of triangular fins is nonmonotonic and not much significant while it shows monotonically increasing behavior for higher values of $\Omega$.
The effect of increasing triangular fin height is very significant on $\overline{N u}_{e}$ which shows its increasing trend. For $\Omega \geq 500$, almost all $\overline{N u}_{e}$ curves tend to be straight.

\section{Conclusions}

In this work, a DG-FEM based numerical investigation of triangular FDP heat exchanger has been performed. The conclusions are summarized as follows:

(1) The computed local result matches the physics of the conjugate heat transfer problem in triangular finned double pipe heat exchanger and validates the present results.

(2) With increase of the conductivity ratio, the heat convection rate at the outer surface of the inner pipe 
TABLE 1: \% overestimates of Nusselt number $\overline{N u}$ for $\widehat{R}=0.5, \beta=3^{\circ}$, and $\delta=0.1$ with [15].

\begin{tabular}{|c|c|c|c|c|c|c|c|c|c|}
\hline \multirow{2}{*}{$H^{*}$} & \multirow{2}{*}{$M$} & \multicolumn{8}{|c|}{$\Omega$} \\
\hline & & 1 & 10 & 20 & 50 & 100 & 500 & 1000 & 5000 \\
\hline \multirow{6}{*}{0.2} & 4 & 84.8 & 12.1 & 6.7 & 2.9 & 1.5 & 0.3 & 0.2 & 0.0 \\
\hline & 8 & 102.1 & 17.5 & 9.9 & 4.4 & 2.3 & 0.5 & 0.2 & 0.0 \\
\hline & 12 & 117.6 & 21.8 & 12.3 & 5.4 & 2.8 & 0.6 & 0.3 & 0.1 \\
\hline & 16 & 129.6 & 25.1 & 14.3 & 6.4 & 3.3 & 0.7 & 0.3 & 0.1 \\
\hline & 20 & 138.0 & 26.0 & 14.5 & 6.3 & 3.3 & 0.7 & 0.3 & 0.1 \\
\hline & 24 & 144.2 & 26.7 & 14.9 & 6.4 & 3.3 & 0.7 & 0.3 & 0.1 \\
\hline \multirow{6}{*}{0.4} & 4 & 117.8 & 26.3 & 16.5 & 8.1 & 4.4 & 0.9 & 0.5 & 0.1 \\
\hline & 8 & 191.0 & 53.3 & 34.0 & 16.7 & 9.1 & 2.0 & 1.0 & 0.2 \\
\hline & 12 & 250.8 & 71.5 & 44.6 & 21.4 & 11.5 & 2.5 & 1.2 & 0.3 \\
\hline & 16 & 288.4 & 80.0 & 48.7 & 22.8 & 12.2 & 2.6 & 1.3 & 0.3 \\
\hline & 20 & 307.9 & 83.8 & 53.1 & 27.1 & 15.4 & 3.5 & 1.8 & 0.4 \\
\hline & 24 & 317.8 & 78.9 & 46.3 & 21.0 & 11.1 & 2.3 & 1.2 & 0.2 \\
\hline \multirow{6}{*}{0.6} & 4 & 150.5 & 41.4 & 27.5 & 14.3 & 8.1 & 1.8 & 0.9 & 0.2 \\
\hline & 8 & 333.8 & 110.7 & 73.6 & 37.8 & 21.1 & 4.7 & 2.4 & 0.5 \\
\hline & 12 & 531.5 & 172.9 & 110.9 & 54.8 & 29.9 & 6.5 & 3.3 & 0.7 \\
\hline & 16 & 671.1 & 206.8 & 128.4 & 61.2 & 32.8 & 7.0 & 3.5 & 0.7 \\
\hline & 20 & 744.2 & 216.9 & 132.4 & 62.4 & 33.4 & 7.1 & 3.6 & 0.7 \\
\hline & 24 & 774.3 & 214.3 & 129.4 & 60.7 & 32.5 & 6.9 & 3.5 & 0.7 \\
\hline \multirow{6}{*}{0.8} & 4 & 170.1 & 50.3 & 34.1 & 18.0 & 10.2 & 2.3 & 1.2 & 0.2 \\
\hline & 8 & 459.4 & 157.8 & 105.0 & 54.0 & 30.2 & 6.7 & 3.4 & 0.7 \\
\hline & 12 & 916.8 & 300.7 & 192.4 & 95.1 & 52.1 & 11.4 & 5.8 & 1.2 \\
\hline & 16 & 1460.2 & 446.2 & 275.9 & 131.6 & 70.9 & 15.2 & 7.7 & 1.5 \\
\hline & 20 & 2019.6 & 574.3 & 347.2 & 162.3 & 86.6 & 18.4 & 9.3 & 1.9 \\
\hline & 24 & 2482.4 & 665.9 & 405.2 & 202.0 & 116.2 & 28.2 & 14.6 & 3.0 \\
\hline
\end{tabular}

between two consecutive fins and at the upper part of the triangular fin surface also increases.

(3) Nusselt number, in general, increases with the conductivity ratio values for the specified values of all the other configuration parameters considered in this work. However, the effect of these parameters is strongly dependent on the conductivity ratio. Therefore, for better design of the triangular finned annulus, these parameters must be chosen in view of the thermal resistance of the material of the inner pipe wall-fin assembly.

(4) The validity of the assumption of infinite conductivity or negligible thermal resistance strongly depends on the height and number of fins and the conductivity ratio. Therefore, in view of the computed results, this assumption should be carefully employed.

(5) The assumption of negligible/zero inner pipe wall thickness can safely be employed for $\Omega \geq 500$.

(6) The Nusselt number based on the equivalent diameter also depicts its strong dependency on the conductivity ratio for $\Omega \leq 500$. However, for $\Omega \geq 500, \overline{N u}_{e}$ does not express substantial rise except for a few cases of a larger number of higher triangular fins.

\section{Nomenclature}

$r_{\mathrm{o}}: \quad$ Inner radius of the outer pipe, $\mathrm{m}$

$r_{\mathrm{i}}$ : $\quad$ Outer radius of the inner pipe, $m$

$r_{1}$ : Radius equal to fin height from the centre of the inner pipe, $\mathrm{m}$

$(r, \phi, z)$ : Cylindrical coordinates, $\mathrm{m}, \mathrm{rad}$, and $\mathrm{m}$

$R: \quad$ Radius, dimensionless

$p_{h}^{*}$ : $\quad$ Heated parameter, dimensionless

$D_{H}^{*}$ : Hydraulic diameter, dimensionless

$D_{e}^{*}: \quad$ Equivalent diameter, dimensionless

$\tau_{b}^{*}: \quad$ Bulk mean fluid temperature,

dimensionless

$p_{e}^{*}$ : $\quad$ Heated parameter based on equivalent diameter, dimensionless

$U_{\max }: \quad$ Maximum fluid velocity, $\mathrm{m} / \mathrm{s}$

$R_{m}: \quad$ Radial point of maximum velocity, $\mathrm{m}$

$\widehat{R}: \quad$ Ratio radii, dimensionless. 

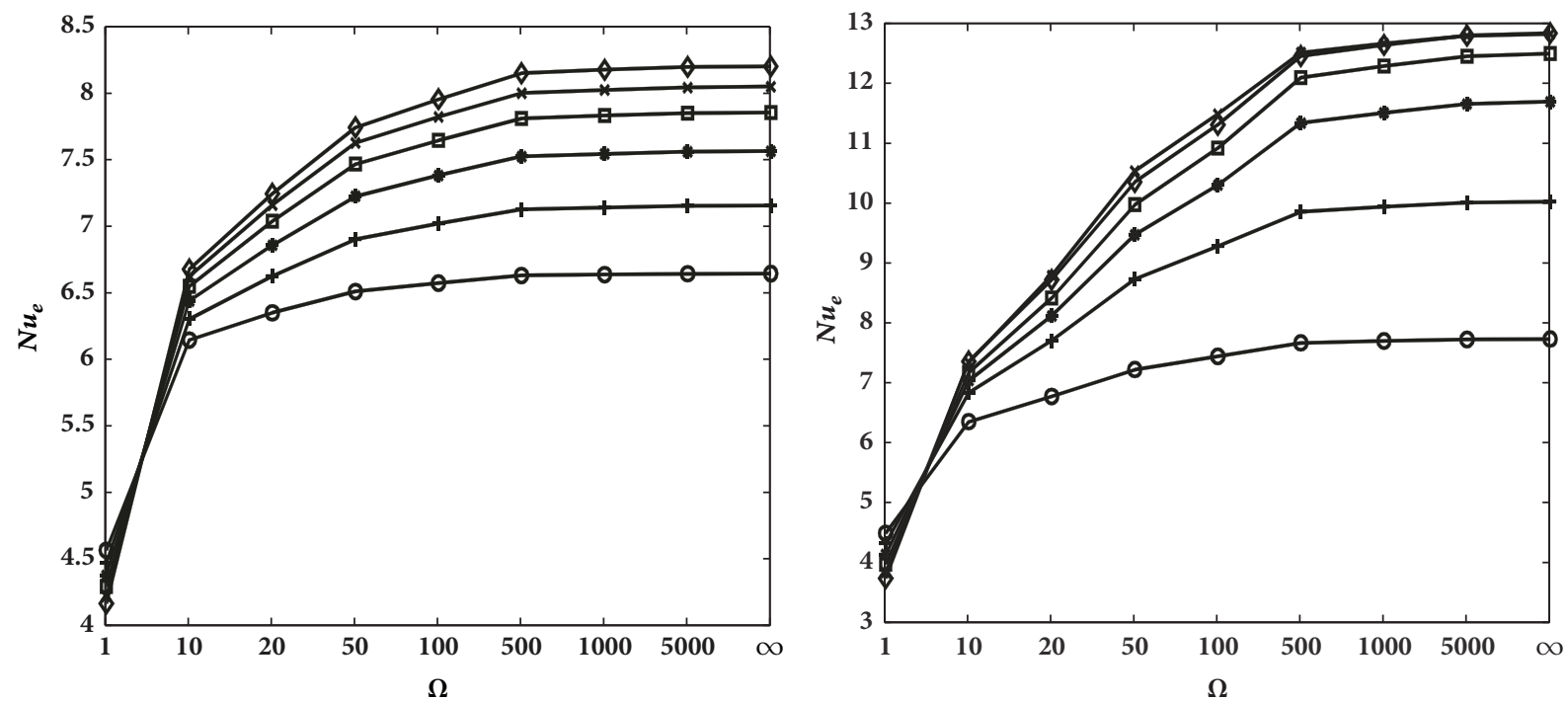

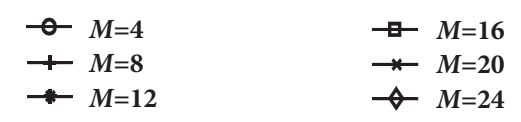

(a) $H^{*}=0.2$
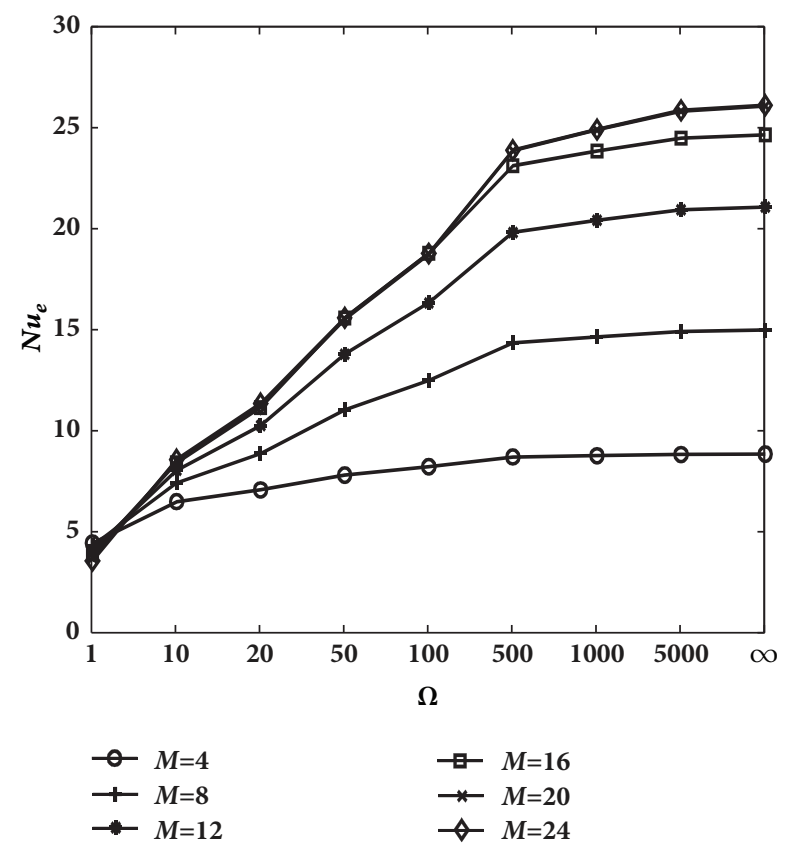

(c) $H^{*}=0.6$

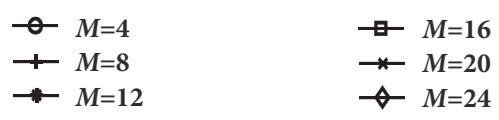

(b) $H^{*}=0.4$

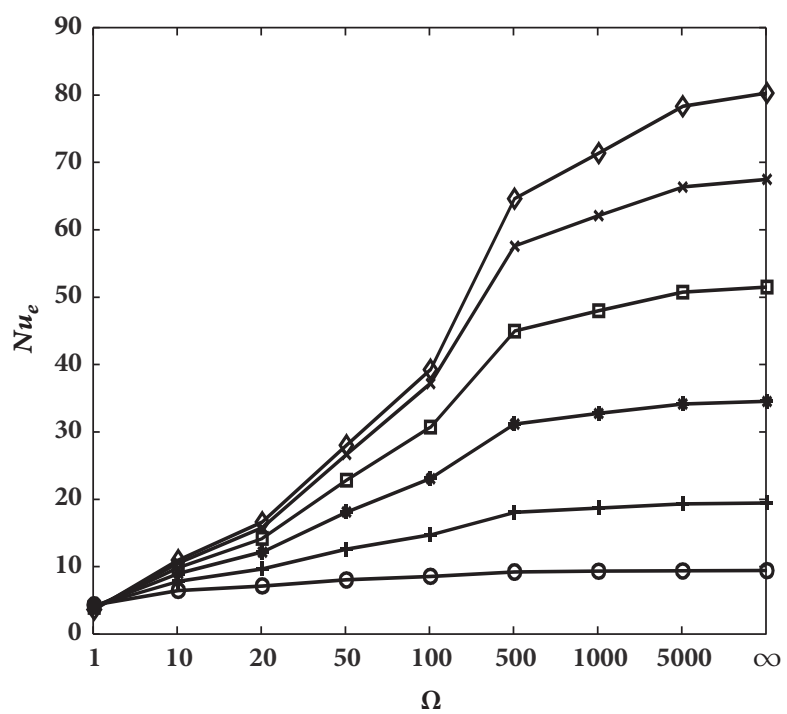

$-M=4$
$+M=8$
$-M=12$

(d) $H^{*}=0.8$

FIGURE 9: $\overline{N u}_{e}$ is plotted against $\Omega$ at $\widehat{R}=0.5, \beta=3^{\circ}$, and $\delta=0.05$.

\section{Data Availability}

The data used to support the findings of this study are available from the corresponding author upon request.

\section{Disclosure}

The present work is based on chapter 6 of the Ph.D. thesis work of the corresponding author, cited in [20].

\section{Conflicts of Interest}

The authors declare that they have no conflicts of interest.

\section{Acknowledgments}

Thanks are due to Higher Education Commission (HEC), Government of Pakistan, for financial support. 


\section{References}

[1] H. M. Soliman, "The effect of fin conductance on laminar heat transfer characteristics of internally finned tubes," The Canadian Journal of Chemical Engineering, vol. 59, no. 2, pp. 251-256, 1981.

[2] B. Krishan, "On conjugated heat transfer in fully developed flow," International Journal of Heat and Mass Transfer, vol. 25, no. 2, pp. 288-289, 1982.

[3] G. S. Barozzi and G. Pagliarini, "A method to solwe conjugate heat transfer problems: The case of fully developed laminar flow in a pipe," Journal of Heat Transfer, vol. 107, no. 1, pp. 77-83, 1985.

[4] M. Sakakibara, S. Mori, and A. Tanimoto, "Conjugate heat transfer with laminar flow in an annulus," The Canadian Journal of Chemical Engineering, vol. 65, no. 4, pp. 541-549, 1987.

[5] W.-Q. Tao, "Conjugated laminar forced convective heat transfer from internally finned tubes," Journal of Heat Transfer, vol. 109, no. 3, pp. 791-795, 1987.

[6] G. Pagliarini, "Effects of axial conduction in the wall and the fluid on conjugate heat transfer in thick-walled circular tubes," International Communications in Heat and Mass Transfer, vol. 15, no. 5, pp. 581-591, 1988.

[7] A. K. Agrawal and S. Sengupta, "Laminar flow and heat transfer in a finned tube annulus," International Journal of Heat and Fluid Flow, vol. 11, no. 1, pp. 54-59, 1990.

[8] I. J. Kettner, D. Degani, and C. Gutfinger, "Numerical study of laminar heat transfer in internally finned tubes," Numerical Heat Transfer, Part A: Applications, vol. 20, no. 2, pp. 159-180, 1991.

[9] N. V. Suryanarayana and T. V. V. R. Apparao, "Heat transfer augmentation and pumping power in double-pipe heat exchangers," Experimental Thermal and Fluid Science, vol. 9, no. 4, pp. 436-444, 1994.

[10] M. Fiebig, Y. Chen, A. Grosse-Gorgemann, and N. K. Mitra, "Conjugate heat transfer of a finned tube part b: Heat transfer augmentation and avoidance of heat transfer reversal by longitudinal vortex generators," Numerical Heat Transfer, Part A: Applications, vol. 28, no. 2, pp. 147-155, 1995.

[11] M. Fiebig, A. Grosse-Gorgemann, Y. Chen, and N. K. Mitra, "Conjugate heat transfer of a finned tube part a: Heat transfer behavior and occurrence of heat transfer reversal," Numerical Heat Transfer, Part A: Applications, vol. 28, no. 2, pp. 133-146, 1995.

[12] J. Taborek, "Double-pipe and multitube heat exchangers with plain and longitudinal finned tubes," Heat Transfer Engineering, vol. 18, no. 2, pp. 34-45, 1997.

[13] K. S. Syed, Simulation of fluid flow through a double-pipe heat exchanger [Ph.D. thesis], Department of Mathematics University of Bradford, 1997.

[14] A. Dorfman and Z. Renner, "Conjugate problems in convective heat transfer: review," Mathematical Problems in Engineering, vol. 2009, Article ID 927350, 27 pages, 2009.

[15] K. S. Syed, M. Ishaq, and M. Bakhsh, "Laminar convection in the annulus of a double-pipe with triangular fins," Computers \& Fluids, vol. 44, no. 1, pp. 43-55, 2011.

[16] Z. Iqbal, K. S. Syed, and M. Ishaq, "Optimal convective heat transfer in double pipe with parabolic fins," International Journal of Heat and Mass Transfer, vol. 54, no. 25-26, pp. 54155426, 2011.

[17] M. Ishaq, K. S. Syed, Z. Iqbal, A. Hassan, and A. Ali, "DGFEM based simulation of laminar convection in an annulus with triangular fins of different heights," International Journal of Thermal Sciences, vol. 72, pp. 125-146, 2013.
[18] Z. Iqbal, K. S. Syed, and M. Ishaq, "Optimal fin shape in finned double pipe with fully developed laminar flow," Applied Thermal Engineering, vol. 51, no. 1-2, pp. 1202-1223, 2013.

[19] S. D. Salman, A. A. H. Kadhum, M. S. Takriff, and A. B. Mohamad, "CFD simulation of heat transfer and friction factor augmentation in a circular tube fitted with elliptic-cut twisted tape inserts," Mathematical Problems in Engineering, vol. 2013, Article ID 163839, 7 pages, 2013.

[20] I. Muhammad, Fem Based Numerical Solutions of Incompressible Flows in a Finned Double Pipe [Ph.D. thesis], Centre for Advanced Studies in Pure and Applied Mathematics (CASPAM), Bahauddin Zakariya University Multan Pakistan, 2013.

[21] Z. Iqbal, M. Ishaq, and K. S. Syed, "Optimization of laminar convection on the shell-side of double pipe with triangular fins," Arabian Journal for Science and Engineering, vol. 39, no. 3, pp. 2307-2321, 2014.

[22] K. S. Syed, M. Ishaq, Z. Iqbal, and A. Hassan, "Numerical study of an innovative design of a finned double-pipe heat exchanger with variable fin-tip thickness," Energy Conversion and Management, vol. 98, pp. 69-80, 2015.

[23] W. Ahmad, K. S. Syed, M. Ishaq, A. Hassan, and Z. Iqbal, "Numerical study of conjugate heat transfer in a doublepipe with exponential fins using DGFEM," Applied Thermal Engineering, vol. 111, pp. 1184-1201, 2017.

[24] J. S. Hesthaven and T. Warburton, Nodal Discontinuous Galerkin Methods Algorithms, Analysis and Applications, Springer, New York, NY, USA, 2008. 


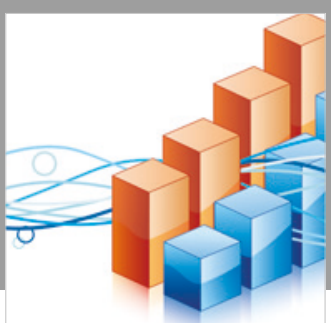

Advances in

Operations Research

\section{-n-m}
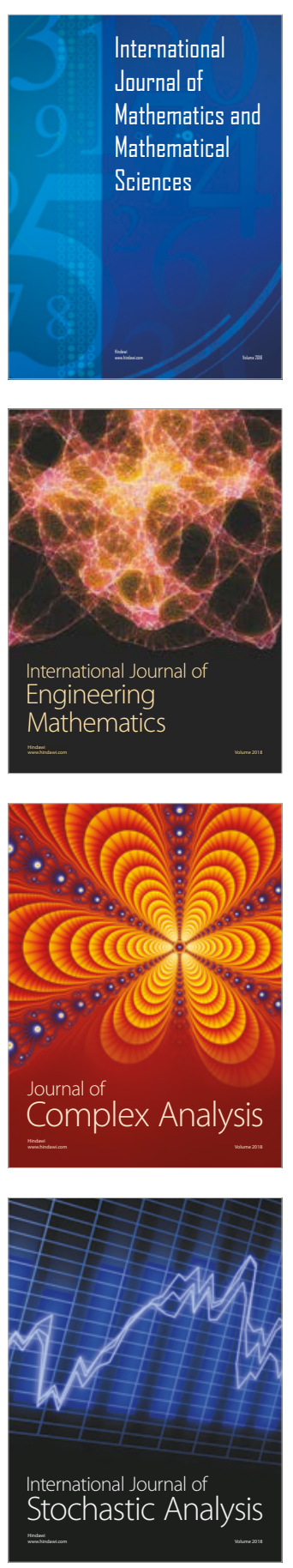
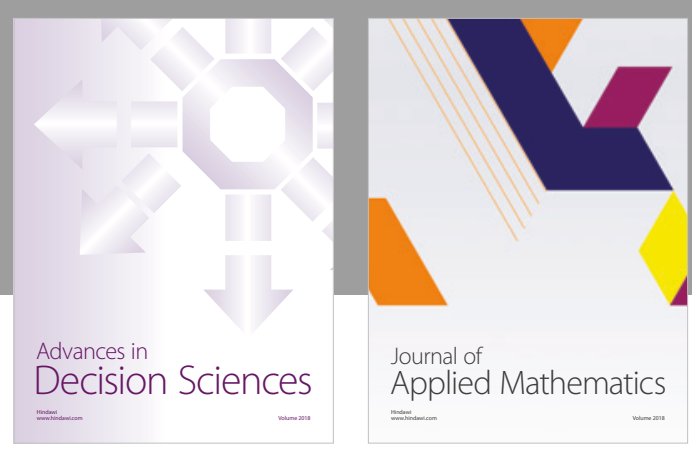

Journal of

Applied Mathematics
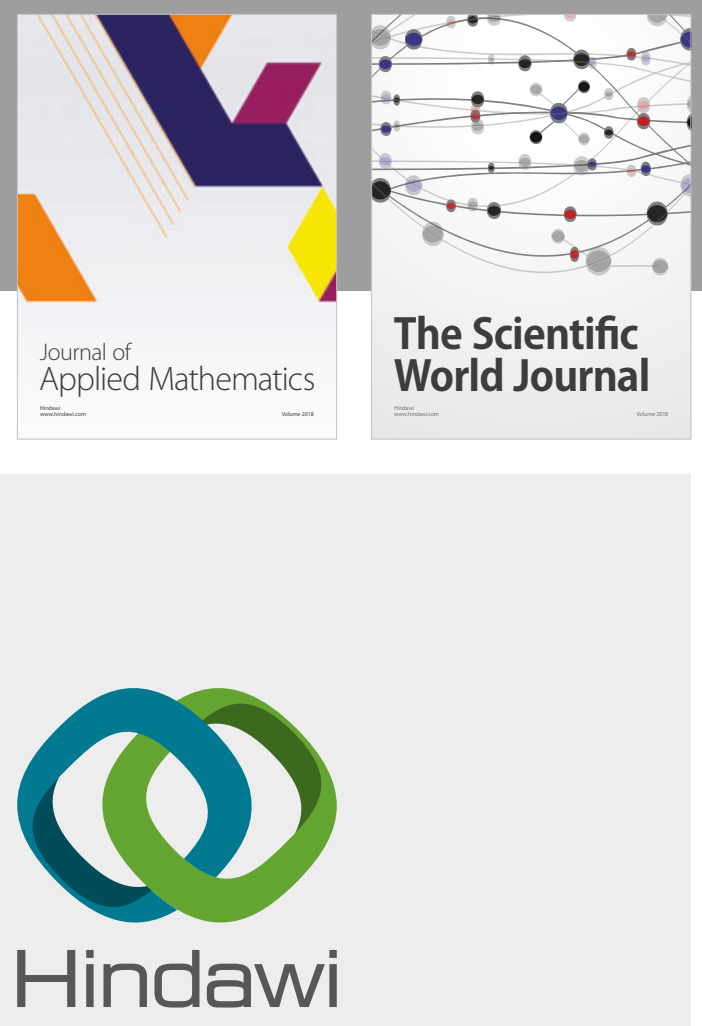

Submit your manuscripts at

www.hindawi.com

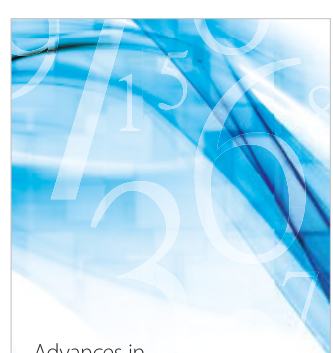

Advances in
Numerical Analysis
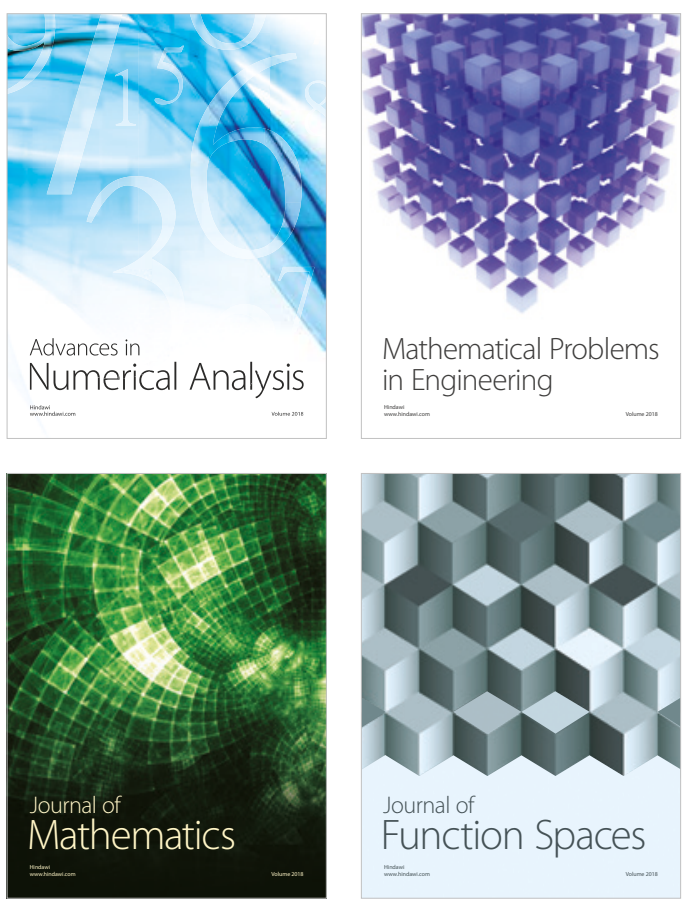

Mathematical Problems in Engineering

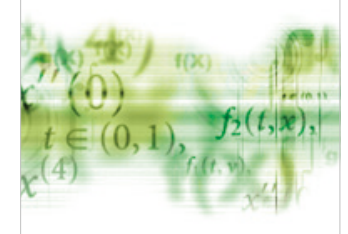

International Journal of

Differential Equations

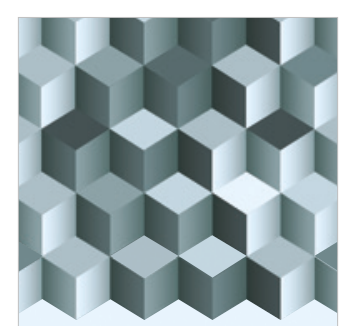

Journal of

Function Spaces

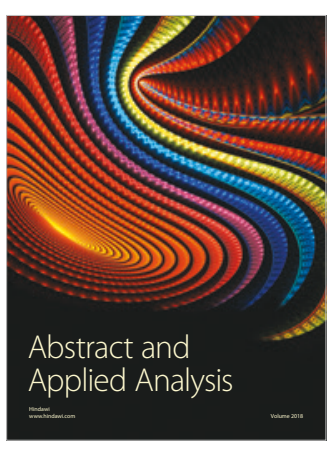

The Scientific

World Journal

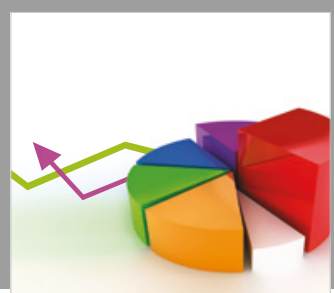

Journal of

Probability and Statistics
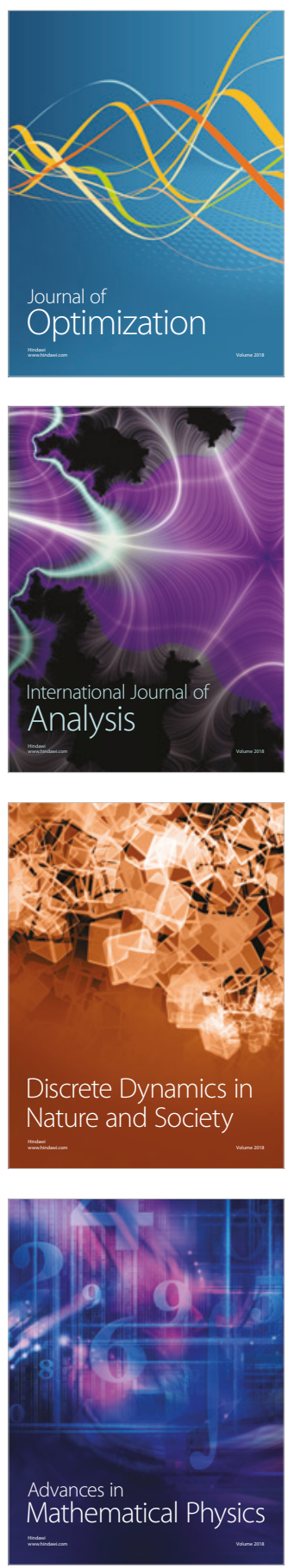